\title{
Alternans and higher-order rhythms in an ionic model of a sheet of ischemic ventricular muscle
}

\author{
Humberto Arce ${ }^{a)}$ \\ Departamento de Física, Facultad de Ciencias, Universidad Nacional Autónoma de México, \\ Apartado Postal 70-542, 04510 México, Distrito Federal, México \\ Aoxiang $\mathrm{Xu}$ \\ Department of Physiology and Centre for Nonlinear Dynamics in Physiology and Medicine, \\ McGill University, 3655 Drummond Street, Montréal, Québec H3G 1Y6, Canada \\ Hortensia González \\ Departamento de Física, Facultad de Ciencias, Universidad Nacional Autónoma de México, \\ Apartado Postal 70-542, 04510 México, Distrito Federal, México \\ Michael R. Guevara \\ Department of Physiology and Centre for Nonlinear Dynamics in Physiology and Medicine, \\ McGill University, 3655 Drummond Street, Montréal, Québec H3G 1Y6, Canada
}

(Received 21 July 1999; accepted for publication 18 January 2000)

\begin{abstract}
Life-threatening arrhythmias such as ventricular tachycardia and fibrillation often occur during acute myocardial ischemia. During the first few minutes following coronary occlusion, there is a gradual rise in the extracellular concentration of potassium ions $\left(\left[\mathrm{K}^{+}\right]_{0}\right)$ within ischemic tissue. This elevation of $\left[\mathrm{K}^{+}\right]_{0}$ is one of the main causes of the electrophysiological changes produced by ischemia, and has been implicated in inducing arrhythmias. We investigate an ionic model of a 3 $\mathrm{cm} \times 3 \mathrm{~cm}$ sheet of normal ventricular myocardium containing an ischemic zone, simulated by elevating $\left[K^{+}\right]_{0}$ within a centrally-placed $1 \mathrm{~cm} \times 1 \mathrm{~cm}$ area of the sheet. As $\left[K^{+}\right]_{0}$ is gradually raised within the ischemic zone from the normal value of $5.4 \mathrm{mM}$, conduction first slows within the ischemic zone and then, at higher $\left[\mathrm{K}^{+}\right]_{0}$, an arc of block develops within that area. The area distal to the arc of block is activated in a delayed fashion by a retrogradely moving wavefront originating from the distal edge of the ischemic zone. With a further increase in $\left[\mathrm{K}^{+}\right]_{0}$, the point eventually comes where a very small increase in $\left[K^{+}\right]_{0}(0.01 \mathrm{mM})$ results in the abrupt transition from a global period-1 rhythm to a global period-2 rhythm in the sheet. In the peripheral part of the ischemic zone and in the normal area surrounding it, there is an alternation of action potential duration, producing a 2:2 response. Within the core of the ischemic zone, there is an alternation between an action potential and a maintained small-amplitude response $(\sim 30 \mathrm{mV}$ in height). With a further increase of $\left[\mathrm{K}^{+}\right]_{0}$, the maintained small-amplitude response turns into a decrementing subthreshold response, so that there is 2:1 block in the central part of the ischemic zone. A still further increase of $\left[K^{+}\right]_{0}$ leads to a transition in the sheet from a global period-2 to a period- 4 rhythm, and then to period- 6 and period- 8 rhythms, and finally to a complete block of propagation within the ischemic core. When the size of the sheet is increased to $4 \mathrm{~cm} \times 4 \mathrm{~cm}$ (with a $2 \mathrm{~cm} \times 2 \mathrm{~cm}$ ischemic area), one observes essentially the same sequence of rhythms, except that the period- 6 rhythm is not seen. Very similar sequences of rhythms are seen as $\left[\mathrm{K}^{+}\right]_{0}$ is increased in the central region (1 or $\left.2 \mathrm{~cm} \mathrm{long}\right)$ of a thin strand of tissue ( 3 or $4 \mathrm{~cm}$ long) in which propagation is essentially one-dimensional and in which retrograde propagation does not occur. While reentrant rhythms resembling tachycardia and fibrillation were not encountered in the above simulations, well-known precursors to such rhythms (e.g., delayed activation, arcs of block, two-component upstrokes, retrograde activation, nascent spiral tips, alternans) were seen. We outline how additional modifications to the ischemic model might result in the emergence of reentrant rhythms following alternans. (C) 2000 American Institute of Physics. [S1054-1500(00)00102-6]
\end{abstract}

\footnotetext{
a) Address for reprints: H. Arce, Departamento de Física, Facultad de Ciencias, Universidad Nacional Autónoma de México, Apartado Postal 70-542, 04510 México, Distrito Federal, México. Electronic mail: jhar@hp.fciencias.unam.mx
} 
Heart attack, a leading cause of death in the industrialized world, is most often caused by a reduction in the flow of blood to the heart muscle ("myocardial ischemia"), typically as a result of blockage of one or more of the coronary arteries ("coronary occlusion'). When the ischemic episode is sufficiently long and intense, cardiac muscle dies ("'myocardial infarction"). However, an individual can sometimes die within the first few minutes following the onset of ischemia ("sudden cardiac death"), before heart cells have a chance to die. Death in this situation is due to a disturbance in the rhythm of the heartbeat ("cardiac arrhythmia") that renders the pumping action of the ventricles ineffective. It has been known for many years that during acute myocardial ischemia this phase of malignant reentrant ventricular arrhythmias (especially ventricular tachycardia and fibrillation) is often preceded by a phase in which there is a beat-to-beat alternation in the electrical activity of the heart ("electrical alternans"). It has been suggested that alternans is the result of a supercritical period-doubling bifurcation, while fibrillation might be chaotic behavior resulting from a subsequent cascade of period-doubling bifurcations as the ischemia becomes more profound. However, the exact nature of any causal relationship between alternans and fibrillation has not yet been demonstrated. Due to the rapid progression of events during ongoing myocardial ischemia, it is very difficult to sort things out in experimental work. We have therefore used numerical simulations with a spatially-distributed ionic model of ischemic ventricular muscle to investigate more exactly what happens as ischemia progresses. While we find that alternans and higher-order rhythms occur in the model as ischemia progresses, we have not been able to produce sustained spiral-wave activity corresponding to reentrant arrhythmias such as ventricular tachycardia and fibrillation. Nevertheless, we do see several of the other phenomena that commonly occur just before such arrhythmias make their appearance.

\section{INTRODUCTION}

Beat-to-beat alternation of the morphology of the electrocardiogram, electrogram, or transmembrane potential ("electrical alternans") has consistently been associated with ventricular arrhythmias in several different situations. ${ }^{1,2}$ In particular, there is considerable clinical evidence that alternans is a frequent occurrence during episodes of acute myocardial ischemia. ${ }^{2-13}$ There is also overwhelming experimental evidence for electrical alternans during ischemia. ${ }^{5,6,9,12,14-36}$

The onset of alternans is very rapid, typically occurring within the first few minutes following coronary artery occlusion, and ventricular arrhythmias, including premature beats and ventricular tachycardia and fibrillation, often start soon after the onset of alternans. The close temporal association of alternans with arrhythmias has led to the suggestion that alternans-perhaps of an amplitude too small to be detected by the naked eye on the electrocardiogram - be monitored as a harbinger of the impending arrhythmias. ${ }^{2,5,9,12,13,32,37-40} \mathrm{In}-$ deed, there is now a device commercially available that quantifies the degree of occult alternans present in the electrocardiogram using power spectral analysis in an attempt at risk stratification. ${ }^{41}$

Despite the close temporal relationship of alternans with arrhythmias, it is not clear whether there is any direct causal relationship-i.e., whether the alternans is inseparably involved in generating the arrhythmias or is merely an epiphenomenon having little or nothing to do with the induction or maintenance of the arrhythmias. Particularly intriguing in this context is the fact that various alternans rhythms have been associated with supercritical period-doubling bifurcations in several other situations in cardiac electrophysiology not involving ischemia, and that a cascade of such perioddoubling bifurcations can result in chaotic cardiac dynamics. ${ }^{42-51}$ In addition, the marked irregularity of ventricular fibrillation has naturally lead to deliberation on whether or not it might be chaotic, ${ }^{38,52-56}$ perhaps being a manifestation of multiple-spiral-wave chaos. ${ }^{57-61}$

However, the fundamental mechanisms underlying ischemic alternans are still to a large extent unknown. Because of the somewhat intractable problems inherent in addressing alternans and arrhythmias in experimental work (lack of stationarity and reproducibility of phenomena during ongoing acute myocardial ischemia, difficulty of recording transmembrane potential from many sites on a fine spatial scale without artifact, inability to record intercellular flows of current), we decided to carry out a numerical study using an ionic model of ventricular muscle from which these drawbacks are expressly excluded.

\section{METHODS}

Ischemic ventricular arrhythmias are caused by alterations in the normal electrophysiological properties of ventricular muscle. These changes include depolarization of the resting membrane potential, fall in the overshoot potential and action potential amplitude, shortening of the action potential duration, slowing of the upstroke velocity and the conduction velocity, and the appearance of post-repolarization refractoriness (for reviews see Refs. 62, 63). These abnormalities are caused by the effects on ionic currents of several disturbances produced by ischemia: e.g., hypoxia, acidosis, increased extracellular potassium concentration. ${ }^{62-64}$ Perhaps the single most important factor is the increase in extracellular potassium concentration $\left(\left[K^{+}\right]_{0}\right)$ which can go from the normal value of $\sim 4-5 \mathrm{mM}$ to as high as $\sim 15 \mathrm{mM}$ in the core of the ischemic zone within the first few minutes following coronary occlusion. ${ }^{65,66}$ At all sites within the ischemic zone, there is a high correlation between the degree of electrophysiological impairment and the local rise in $\left[\mathrm{K}^{+}\right]_{0}$ (e.g., compare Fig. 4 in Ref. 67 with Figs. 7 and 8 in that paper). In addition, regional coronary artery perfusion with a high $\mathrm{K}^{+}$solution mimics much of the behavior seen during acute myocardial ischemia, including alternans and ventricular arrhythmias. ${ }^{66,68,69} \mathrm{We}$ decided therefore, as in previous work, ${ }^{70}$ to model the ischemic ventricle 
TABLE I. Periodicity of rhythms as function of $\left[K^{+}\right]_{0}(\mathrm{mM})$

\begin{tabular}{ccc}
\hline \hline Period & $\begin{array}{c}\text { Sheet } \\
(3 \mathrm{~cm} \times 3 \mathrm{~cm})\end{array}$ & $\begin{array}{c}\text { Strand } \\
(3 \mathrm{~cm} \times 0.125 \mathrm{~cm})\end{array}$ \\
\hline 1 & $9.00-13.39$ & $9.000-13.104$ \\
2 & $13.40-13.69$ & $13.105-13.380$ \\
4 & $13.70-13.72$ & $13.400-13.430$ \\
6 & $13.729-13.733$ & $13.435-13.450$ \\
8 & 13.734 & 13.455 \\
Complete block & $13.735-14.000$ & $13.460-14.000$ \\
\hline \hline
\end{tabular}

as a two-dimensional sheet of normal myocardium with an area of elevated $\left[K^{+}\right]_{0}$ embedded within its interior to represent the ischemic zone.

The first step in modeling is to choose a particular ionic model of normal ventricular muscle from the many models now available (see, e.g., the list in Ref. 70). We have avoided using one of the more recent "second-generation" models that have the sodium-potassium pump, the sodium-calcium exchanger, and internal calcium handling since there are complications with this class of models involving long-term drifts of ionic concentrations ${ }^{71-73}$ and degeneracy of equilibria. $^{74,75}$ We have selected the Luo and Rudy (LR) first-generation model ${ }^{76}$ largely because it has $\left[K^{+}\right]_{0}$ as a parameter, which is essential for our modeling of the ischemic zone.

One deficiency of the LR model, which is carried over from the Beeler-Reuter model from which it was derived, is that one can obtain unphysiologic spike-like action potentials of extremely brief duration at a relatively long diastolic interval when the stimulus amplitude is low. This artifact occurs because the time constant for activation of the calcium current $\left(I_{s}\right)$ is about an order of magnitude too large in the LR model. To minimize this artifact, which can manifest itself when excitability is low in an area of elevated $\left[\mathrm{K}^{+}\right]_{0}$, we have decreased the time constant for the activation of $I_{s}$ $\left(\tau_{d}\right)$ by a factor of 10 , which then puts it into the physiologic range. $^{70,77}$ This modification reduces the action potential duration (measured from upstroke to $-60 \mathrm{mV}$ on the repolarizing limb of the action potential) in the standard LR spaceclamped model when paced at a basic cycle length of $400 \mathrm{~ms}$ from $\sim 290 \mathrm{~ms}$ to $\sim 237 \mathrm{~ms}$, which is within the range of values encountered in normal ventricular tissue paced at that basic cycle length in human beings ${ }^{78-80}$ and in the species most commonly used in experimental work on ischemic arrhythmias. ${ }^{23,29,30} \mathrm{We}$ have used the equations appearing in Table I and the body of the text of Luo and Rudy (1991), ${ }^{76}$ together with $R T / F=26.5 \mathrm{mV}$.

We model a two-dimensional sheet of isotropic ventricular muscle by the two-dimensional cable equation,

$$
\left(\frac{\partial^{2} V}{\partial x^{2}}+\frac{\partial^{2} V}{\partial y^{2}}\right)=\rho S_{\nu}\left(C_{m} \frac{\partial V}{\partial t}+I_{\text {ion }}\right),
$$

where $V$ is the transmembrane potential $(\mathrm{mV}), x$ and $y$ are spatial coordinates in the sheet $(\mathrm{cm}), \rho$ is the bulk cytoplasmic resistivity $(0.2 \mathrm{k} \Omega-\mathrm{cm}), S_{\nu}$ is the surface-to-volume ratio $\left(5000 \mathrm{~cm}^{-1}\right), C_{m}$ is the specific membrane capacitance (1 $\mu \mathrm{F} \mathrm{cm}{ }^{-2}$ ), $t$ is time (ms), and $I_{\text {ion }}$ is the total ionic current $\left(\mu \mathrm{A} \mathrm{cm}^{-2}\right.$ ) given by the LR model. We use an explicit integration scheme, with forward Euler integration for the internal calcium concentration, and an exact analytic formula for the activation and inactivation variables (for further details of the integration scheme, see Ref. 70). The temporal integration step-size $(\Delta t)$ is $0.025 \mathrm{~ms}$ and the spatial integration step-size $(\Delta x=\Delta y)$ is $0.025 \mathrm{~cm}^{59}$ The onedimensional space-constant is $\lambda=\left(R_{m} / \rho S_{\nu}\right)^{1 / 2} \cong 0.06 \mathrm{~cm}$, where $R_{m}$ is the specific membrane resistance $\left(3.55 \mathrm{k} \Omega-\mathrm{cm}^{2}\right.$ at the nominal LR value of $\left[K^{+}\right]_{0}$ of $\left.5.4 \mathrm{mM}\right)$. The diffusion constant $D=1 /\left(\rho S_{\nu} C\right)=10^{-3} \mathrm{~cm}^{2} \mathrm{~ms}^{-1}$, so that the von Neumann linear stability criterion $\left[(\Delta x)^{2} / \Delta t>4 D\right]$ is satisfied. This integration scheme results in a planar-wave propagation velocity from infinite-rest initial conditions of $55 \mathrm{~cm}$ $\mathrm{s}^{-1}$, which increases by $13 \%$ to $62 \mathrm{~cm} \mathrm{~s}^{-1}$ when a 2.5 fold finer space-time discretization grid is used $(\Delta t=0.01 \mathrm{~ms}$ and $\Delta x=\Delta y=0.01 \mathrm{~cm}$ ). The maximum upstroke velocity from infinite-rest initial conditions is $343 \mathrm{~V} \mathrm{~s}^{-1}$. Simulations were carried out on Pentium Pro (200 MHz) and SiliconCray Origin 2000 machines using programs written in $\mathrm{C}$ ( $\sim 16$ significant decimal places $)$.

\section{RESULTS}

We investigate the propagation of the action potential in a $3 \mathrm{~cm} \times 3 \mathrm{~cm}$ square of ventricular muscle $(120 \times 120 \mathrm{nu}-$ merical grid) with a centrally placed $1 \mathrm{~cm} \times 1 \mathrm{~cm}$ ischemic zone (grid-points 40 to 80 in both directions). Stimulation of this quiescent sheet is provided by injecting a periodic train of current pulses (amplitude $=300 \mu \mathrm{A} \mathrm{cm}{ }^{-2} \cong$ twice diastolic threshold, duration $=1 \mathrm{~ms}$ ) into the column of elements along the left-hand edge of the sheet at a basic cycle length (BCL) of $400 \mathrm{~ms}$, which is in the middle of the range of pacing intervals typically employed in experimental work on ischemic arrhythmias (300-500 ms). ${ }^{18,21,29-32,37}$ A series of simulation runs was made, with $\left[\mathrm{K}^{+}\right]_{0}$ in the ischemic area being increased on each new run. Initial conditions were obtained at each new $\left[K^{+}\right]_{0}$ by setting the variables in both the normal and ischemic areas equal to their corresponding space-clamped steady-state values and then allowing 1000 $\mathrm{ms}$ to pass to allow time for equilibration towards the asymptotic steady-state rest condition, at which time the first stimulus pulse was injected.

\section{A. 1:1 rhythm with orthograde propagation}

For $\left[K^{+}\right]_{0}$ not too high, the action potential propagates throughout the entire sheet. Figure 1(A) shows the activation map of the transmembrane potential $V$ at four different times with $\left[K^{+}\right]_{0}=12.0 \mathrm{mM}$ in the ischemic zone (dark red corresponds to the most depolarized potential, dark blue to the most hyperpolarized: see the color-bar map at the extreme right in Fig. 1). At $t=10 \mathrm{~ms}$ ( $t=0 \mathrm{~ms}$ corresponds to the start of delivery of the tenth stimulus pulse at $B C L=400 \mathrm{~ms}$ ) the activation wavefront, which is traveling as a plane wave, has not yet reached the ischemic area [the leftmost panel of Fig. 1(A)]. As the wavefront penetrates into the ischemic zone [the centrally-placed slightly depolarized area in light blue in Fig. 1(A)], the conduction velocity falls, causing the activation wavefront to become curved [Fig. 1(A): $t=30 \mathrm{~ms}$ ]. 


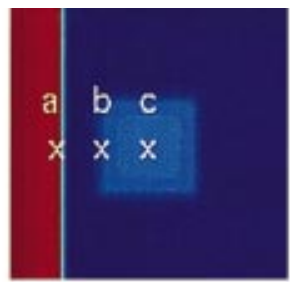

$10 \mathrm{~ms}$

B

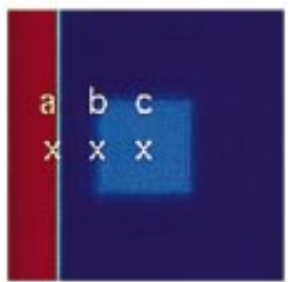

$10 \mathrm{~ms}$

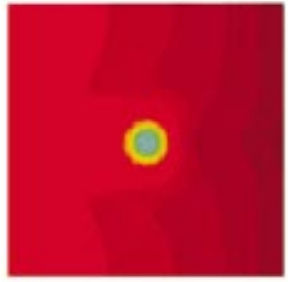

$70 \mathrm{~ms}$
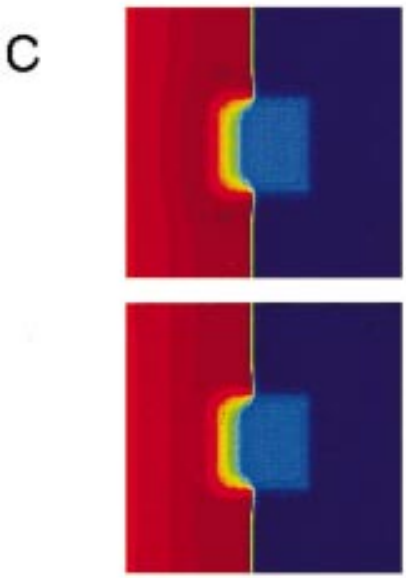

$25 \mathrm{~ms}$

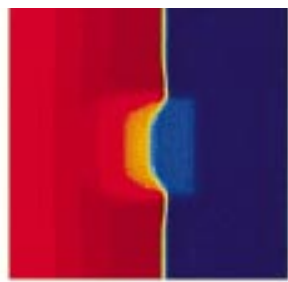

$30 \mathrm{~ms}$

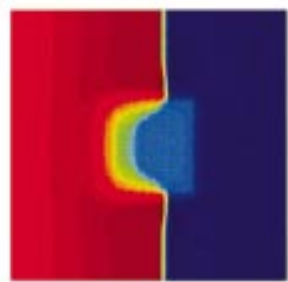

$30 \mathrm{~ms}$

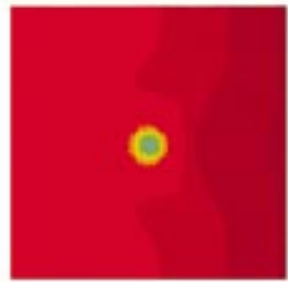

$80 \mathrm{~ms}$
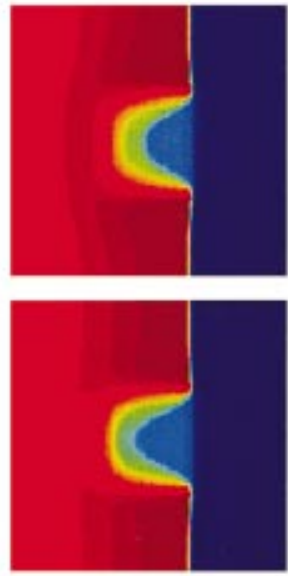

$35 \mathrm{~ms}$

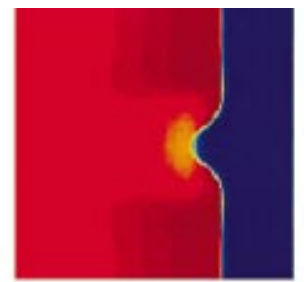

$40 \mathrm{~ms}$

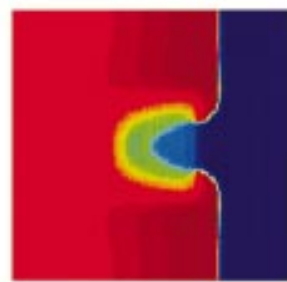

$40 \mathrm{~ms}$

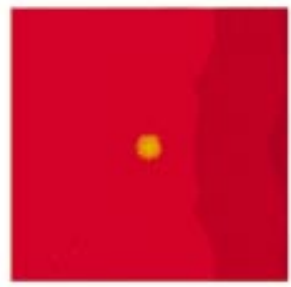

$90 \mathrm{~ms}$
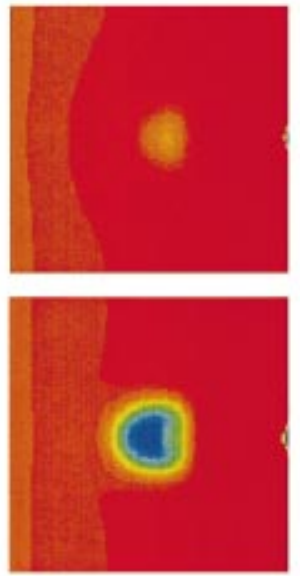

$55 \mathrm{~ms}$

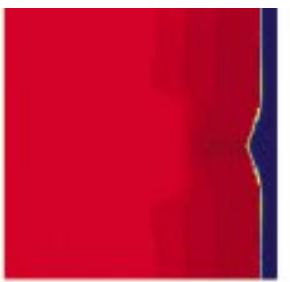

$50 \mathrm{~ms}$

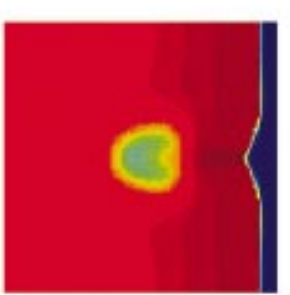

$50 \mathrm{~ms}$

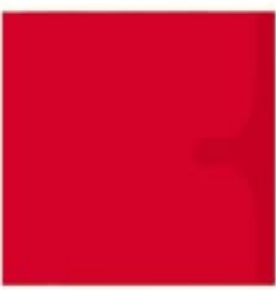

$100 \mathrm{~ms}$
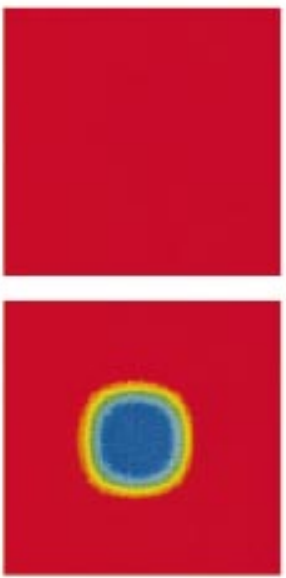

$130 \mathrm{~ms}$

FIG. 1. (Color) (A) 1:1 rhythm with anterograde propagation ( $\left[\mathrm{K}^{+}\right]_{0}=12.0 \mathrm{mM}$ in the ischemic zone). Activation maps (transmembrane voltage as a function of location in the sheet) showing propagation of the tenth action potential at BCL=400 ms. The time indicated for each map is the time following the start of injection of the tenth stimulus pulse. The calibration scale at right is for the transmembrane potential. Propagation speed is decreased in the ischemic zone (the light blue area in the leftmost panel) producing delayed activation with respect to the normal zone. (B) 1:1 rhythm with retrograde propagation $\left(\left[\mathrm{K}^{+}\right]_{0}=13.39 \mathrm{mM}\right.$ in the ischemic zone). Activation maps showing that activation of a part of the distal end of the ischemic zone is markedly delayed. The wavefronts propagating in the normal area above and below the ischemic zone now retrogradely invade it in an encircling movement. (C) Period-2 rhythm $\left(\left[\mathrm{K}^{+}\right]_{0}=13.45 \mathrm{mM}\right.$ in ischemic zone). The activation maps in the upper row pertain to the conducted beat [as in (A), (B)], while those in the lower row pertain to the blocked beat in which the core of the ischemic zone remains unexcited. A repolarization wave expands out from this unexcited area. The rhythm is $2: 1$ in the unexcited core of the ischemic area, and 2:2 outside of this area.

This decrease in conduction velocity is associated with a reduced amplitude and upstroke velocity of the action potential [action potentials $a, b, c$ in Fig. 2(A) are taken from the corresponding locations in Fig. 1(A)]. These two effects are in turn caused by the $K^{+}$-induced depolarization of the resting membrane potential [RMP in Fig. 2(B)] and subsequent resting inactivation of the fast inward sodium current. The increasing depolarization of the resting membrane potential as the proximal edge of the ischemic zone is approached and traversed causes a gradual fall in the overshoot potential (OS), the peak voltage attained by the action potential [Fig. 2(B)]. The action potential amplitude (the difference between OS and RMP) thus also gradually falls in the border zone (a thin annular region spreading out on both sides of the 
A
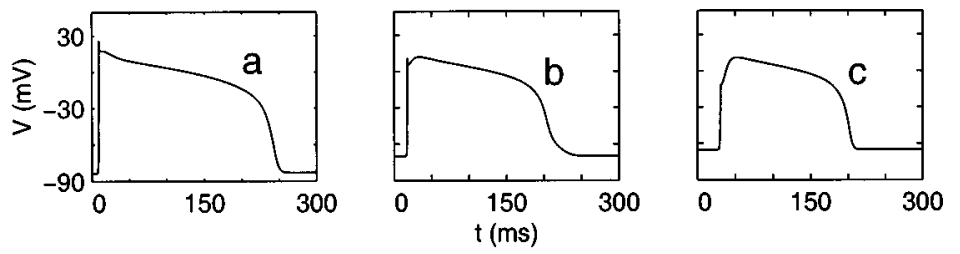

B

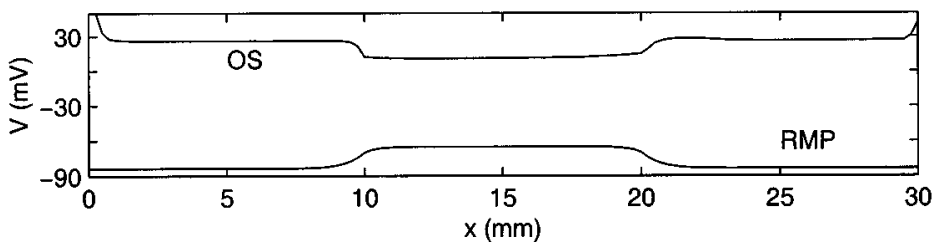

FIG. 2. 1:1 rhythm with anterograde propagation $\left(\left[\mathrm{K}^{+}\right]_{0}=12.0 \mathrm{mM}\right.$ in the ischemic zone). (A) Transmembrane potential versus time from three positions marked by $X$ in the leftmost map in Fig. 1(A) ( $x$ $=0.5,0.975$, and $1.5 \mathrm{~cm}$ for $a, b$, and $c$, respectively). (B) Overshoot potential (OS) and resting membrane potential (RMP) measured along a horizontal line passing through the points $a, b$, and $c$ in Fig. 1(A). normal/ischemic boundary) as the ischemic zone is approached and entered [Fig. 2(B)]. Indeed, one even begins to see two-component upstrokes of the action potential towards the center of the ischemic zone [Fig. 2(A): trace $c$ ]. The action potential duration (APD) also shortens significantly within the ischemic zone [Fig. 2(A)]. However, as the distal edge of the ischemic zone is approached, the reverse process occurs, with a gradual recovery of resting membrane potential, overshoot potential, action potential amplitude, APD, and conduction velocity back to their normal values as the distal border zone is traversed [Fig. 2(B)]. There is therefore decremental conduction as the wavefront invades the ischemic zone and incremental conduction as it leaves.

Thus, for $\left[K^{+}\right]_{0}$ sufficiently small, the sheet as a whole displays a global 1:1 activation pattern, with any given location on the sheet being activated on each beat at a fixed latency ("activation time") following the stimulus pulse. The maximum value of the activation time within the ischemic zone occurs at the central part of the distal edge, where it is $\sim 40 \mathrm{~ms}$ [Fig. $1(\mathrm{~A}): t=40 \mathrm{~ms}$ ].

\section{B. 1:1 rhythm with retrograde propagation}

As $\left[K^{+}\right]_{0}$ is raised further beyond $12 \mathrm{mM}$, the conduction velocity progressively falls within the ischemic zone, but the global 1:1 activation pattern is maintained. However, there eventually comes a point where there is a change in the activation sequence within the ischemic zone. Figure 1(B) shows the situation at $\left[K^{+}\right]_{0}=13.39 \mathrm{mM}$ : there is still a global 1:1 rhythm, but the activation wavefront that had previously successfully propagated through the ischemic zone at a lower $\left[K^{+}\right]_{0}$ [e.g., $12.0 \mathrm{mM}$ in Fig. 1(A)] is blocked within the ischemic zone, creating an arc of conduction block [Fig. 1(B): $t=30 \mathrm{~ms}$ and $t=40 \mathrm{~ms}$ ]. The amplitude of the action potential gradually falls as it travels within the ischemic zone, until only a residual small-amplitude response remains (the first upward deflection in trace $c$ of Fig. 3). An area within the ischemic zone, with its center lying just to the right of the center of the ischemic zone [Fig. 1(B): $t \geqslant 40$ $\mathrm{ms}$, is now activated by two wavefronts that merge and retrogradely invade the ischemic zone across its right-hand border, after traveling initially in the normal myocardium above and below the ischemic zone $[t=40 \mathrm{~ms}$ in Fig. 1(B)]. A third source of stimulation current to this area is the part of the depolarized area surrounding this core that was activated earlier in the orthograde fashion. As time proceeds, this unexcited core area shrinks in size very slowly [Fig. 1(B): bottom row of panels], being activated in a very delayed fashion [maximum activation time of $\sim 100 \mathrm{~ms}$ in Fig. 1(B) versus $\sim 40 \mathrm{~ms}$ in Fig. 1(A)], producing the slowly rising action potential following the small-amplitude response in trace $c$ of Fig. 3. Delayed activation is almost invariably seen during experimental work on ischemia, and is responsible for producing a fractionated electrogram at sites within the ischemic zone. $^{16,19,27,28,30,81,82}$

\section{Alternans}

As $\left[K^{+}\right]_{0}$ is increased further, there is eventually a sudden loss of the global 1:1 activation pattern. For the first time, there are now elements of the sheet that no longer respond to each stimulus with an action potential. The central core of the ischemic zone is activated only on alternate beats, resulting in a local 2:1 response. The two rows of panels in Fig. 1(C) show activation maps on alternate beats at $\left[K^{+}\right]_{0}=13.45 \mathrm{mM}$ (top row: conducted beat; bottom row: blocked beat). On both the conducted and blocked beats, the action potential decrements in amplitude as it enters and traverses the proximal part of the ischemic zone [traces $a, b$ in Fig. 4(A) and Fig. 4(B), respectively], as during 1:1 rhythm [Figs. 1(A), 1(B), 2, 3]. However, there is a greater degree of decrement for the blocked beat than for the conducted beat [compare the two activation maps at $t=35 \mathrm{~ms}$ in Fig. 1(C) and the two action potentials at location $b$ in Figs. 4(A) and 4(B) ]. The action potential on the blocked beat falls
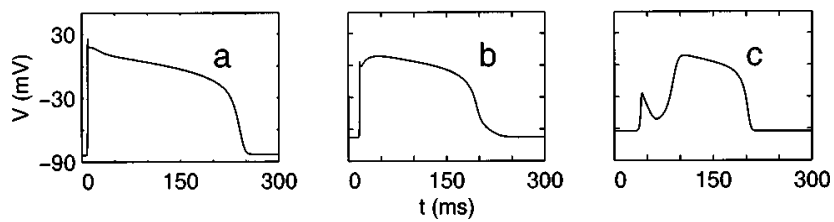

FIG. 3. 1:1 rhythm with retrograde propagation $\left(\left[\mathrm{K}^{+}\right]_{0}=13.39 \mathrm{mM}\right.$ in the ischemic zone). Transmembrane potential at the points indicated in the leftmost panel of Fig. 1(B) [the same locations as in Fig. 1(A)]. The response at site $c$ now exhibits two components: the first is a small-amplitude deflection associated with anterograde propagation, while the second is a very delayed slow-response action potential associated with retrograde propagation. 
A
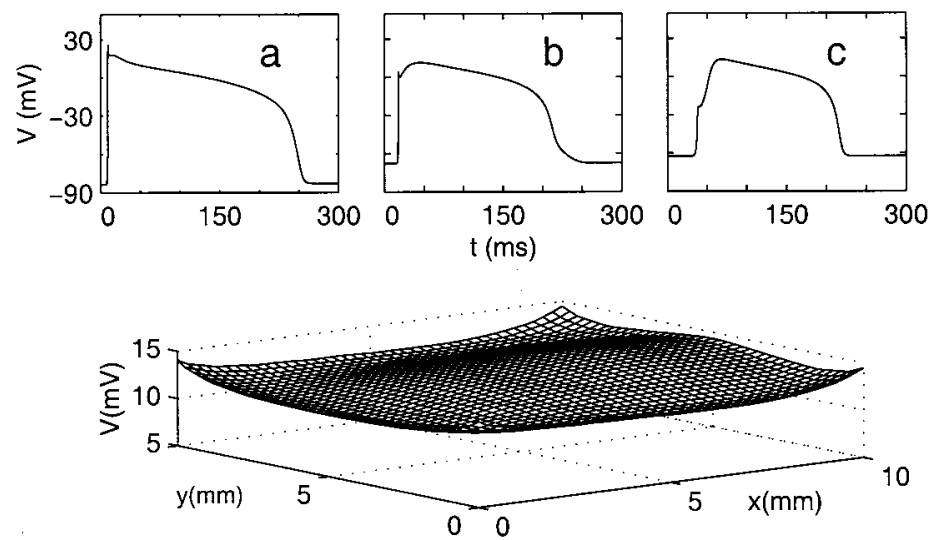

B
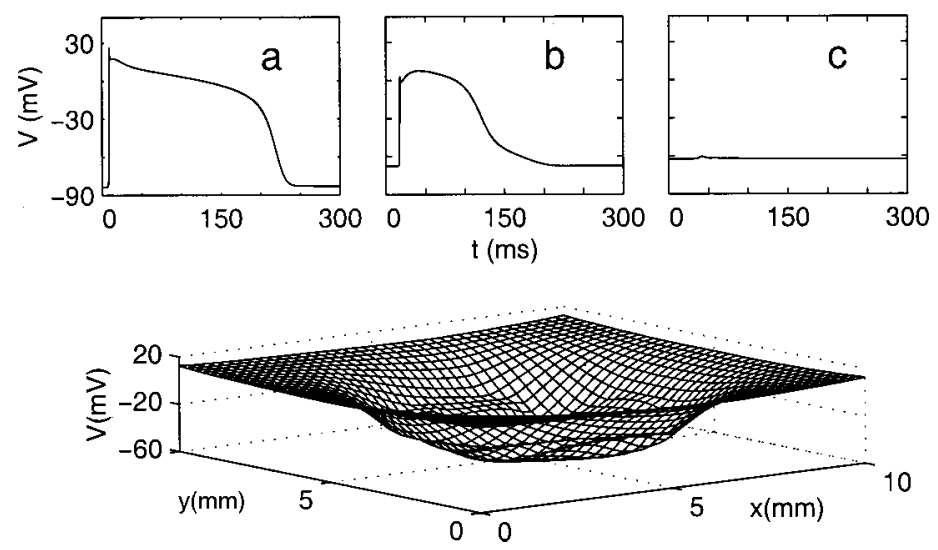

FIG. 4. Period-2 rhythm $\left(\left[\mathrm{K}^{+}\right]_{0}=13.45 \mathrm{mM}\right.$ in the ischemic zone). Action potentials (top row) and maximum voltage map (bottom row) for the conducted beat (A) and the blocked beat (B) of Fig. 1(C). The traces obtained at sites $a$ and $b$ exhibit a 2:2 rhythm (i.e., alternans), which is more obvious at location $b$, which lies in the normal area just to the left of the proximal edge of the ischemic zone [see the leftmost panel in Fig. 1(A) or 1(B) for site locations]. The core of the ischemic zone (site $c$ ) displays a 2:1 rhythm. The maximum voltage map gives the highest voltage reached at each point within the ischemic zone (not the entire sheet) during the $400 \mathrm{~ms}$ of the pacing cycle. There is a $2: 1$ rhythm in the core of the ischemic zone, with a $2: 2$ rhythm in the surrounding area. in size with increasing penetration into the ischemic zone to the point where only a barely noticeable subthreshold electrotonic response remains [trace $c$ in Fig. 4(B)]. Note that this occurs despite the fact that the distal edge of the ischemic zone is again invaded by a wavefront traveling in the retrograde direction, as with the conducted beat. Indeed, during the period of time corresponding to that during which the central area is being activated by an inwardly moving wave of depolarization on the conducted beat [top row of Fig. 1(C): $t=55$ and $130 \mathrm{~ms}$ ], there is in contrast a wave of repolarization propagating away from that area on the blocked beat [bottom row of Fig. 1(C): $t=55$ and $130 \mathrm{~ms}$ ].

The size of the area of 2:1 block is perhaps best appreciated from the "maximum voltage map," which gives the maximum transmembrane voltage (which is the overshoot potential when the response is an action potential) attained during the course of one pacing cycle at each location. Figures 4(A) and 4(B) (bottom panels) show this map for the ischemic zone alone (i.e., not for the entire sheet) on alternate beats. Figure 4(B) (bottom panel) shows that there is a large area of the ischemic zone that remains unexcited on the blocked beat, remaining at its resting potential of about -60 $\mathrm{mV}$, with decremental propagation into that area from the surrounding annular area. As $\left[K^{+}\right]_{0}$ increases in the range over which 2:1 block occurs, there is a growth in the size of this area of $2: 1$ block.

Note that there is a marked beat-to-beat alternation of action potential morphology at location $b$ in Fig. 4. We refer to this as a 2:2 response, since the behavior is periodic in time with the repeating unit consisting of two stimuli and two action potentials of different morphologies. Inspection of the voltage waveform at locations increasingly to the right of location $b$ reveals that the degree of alternation increases, with the smaller of the two action potentials gradually falling in size. Eventually, this action potential is extinguished, terminating in a subthreshold response that decays in size as the center of the ischemic core is approached. At some location that depends on one's arbitrary definition of an action potential, the $2: 2$ response is thus replaced by a $2: 1$ response. Conversely, as one examines locations increasingly to the left of $b$, the degree of alternation gradually becomes less pronounced (see, e.g., location $a$ in Fig. 4). Indeed, $1.25 \mathrm{~mm}$ away from the left edge of the sheet (i.e., $8.75 \mathrm{~mm}$ from the left-hand boundary of the ischemic zone), the difference in APD of the two action potentials is only $8 \mathrm{~ms}$. Thus, a cursory examination of voltage traces at a site sufficiently to the left of location $a$ in a sheet with a large enough normal area surrounding the ischemic area might lead to the misinterpretation that a $1: 1$ response is present, since closer inspection would reveal a very slight degree of alternation. In a sheet of infinite size, one would recover the 1:1 response in the limit of the measurement site being infinitely far away from the ischemic core.

The transition to $2: 1$ block in the ischemic core [Figs. $1(\mathrm{C}), 4]$ occurred abruptly, given that a 1:1 rhythm was seen at a value of $\left[\mathrm{K}^{+}\right]_{0}$ only $0.06 \mathrm{mM}$ lower [Figs. 1(B), 3]. While a direct transition from 1:1 rhythm to $2: 1$ rhythm with no intervening rhythms has been described in periodically 
A

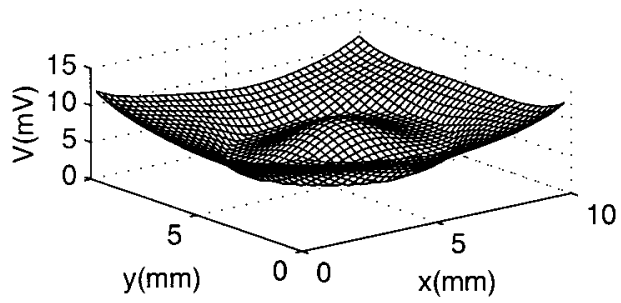

$\mathrm{B}$
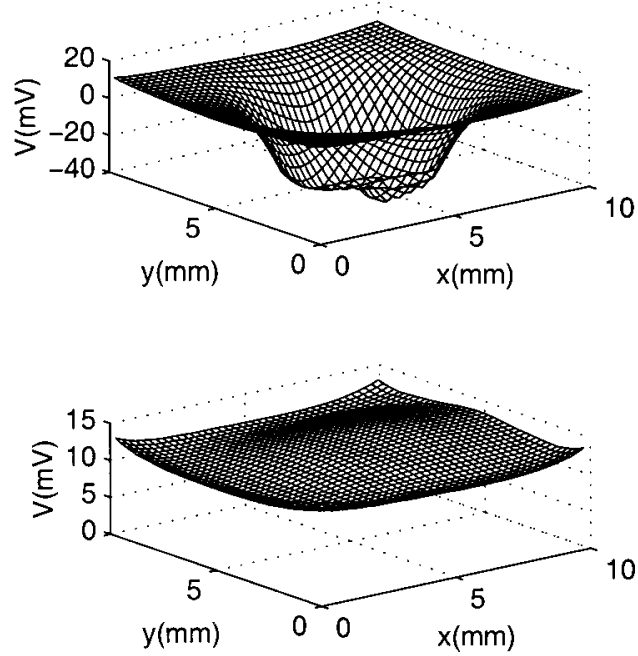

FIG. 5. Transition from period-1 to period-2 rhythm. Maximum voltage maps of the ischemic zone. (A) Period-1 rhythm at $\left[K^{+}\right]_{0}=13.39 \mathrm{mM}$. (B) Period-2 rhythm at $\left[K^{+}\right]_{0}=13.40 \mathrm{mM}$ (the top panel is a maintained smallamplitude response; the bottom panel is the full-sized propagated action potential). Note that while voltage scales are the same in (A) and the bottom panel of (B), a different voltage scale is used in the top panel of (B).

driven single rabbit ventricular cells and in the spaceclamped LR model, ${ }^{83}$ the transition can also be indirect, with Wenckebach $^{84-86}$ or alternans ${ }^{87,88}$ rhythms being seen before the $2: 1$ rhythm makes its appearance. We therefore went back and investigated the transition from period-1 to period-2 rhythm in the sheet more closely. This transition is very abrupt, being seen when $\left[K^{+}\right]_{0}$ is raised by as little as $0.01 \mathrm{mM}$. Figure 5(A) shows the maximum voltage map of the period- 1 rhythm seen at $\left[K^{+}\right]_{0}=13.39 \mathrm{mM}$ [Fig. 1(B)], while Fig. 5(B) shows the maximum voltage map on alternate beats of the period-2 rhythm encountered at $\left[K^{+}\right]_{0}$ $=13.40 \mathrm{mM}$. During this period-2 rhythm, the response in the core of the ischemic area is not a $2: 1$ rhythm, since on alternate beats the maximum voltage in that area is about $-30 \mathrm{mV}$ [the top panel of Fig. 4(B)], and not the resting membrane potential of $\sim-60 \mathrm{mV}$ as in the $2: 1$ response of Fig. 4 (where $\left[K^{+}\right]_{0}$ is only slightly higher at $13.45 \mathrm{mM}$ ). The time-course of the transmembrane potential in the core of the ischemic area on this beat is very similar to that of the first deflection seen in trace $c$ of Fig. 3. While this waveform resembles a subthreshold deflection, and not an action potential, it does not decrement electrotonically with distance as does the classic subthreshold response, but rather is maintained in size and shape over a distance of several space constants [the upper panel of Fig. 5(B)]. We term this response the "maintained small-amplitude response.' Given that this response is not a decrementing one as in Fig. 4(B), one has a 2:2 rhythm in the ischemic core-and everywhere else in the sheet. When $\left[K^{+}\right]_{0}$ is raised to $13.45 \mathrm{mM}$, the $2: 2$ rhythm in the core of the ischemic zone converts into a $2: 1$ rhythm [Figs. 1(C), 4], which persists to $\left[K^{+}\right]_{0}=13.69 \mathrm{mM}$.

Note also that the maximum voltage during 1:1 rhythm (which corresponds to the overshoot potential) just before the transition to period-2 rhythm [Fig. 5(A)] is less than the overshoot potential of the action potential during 2:2 rhythm just after the transition [the lower panel of Fig. 5(B)]. The explanation for this is that the recovery time or diastolic interval preceding the longer duration action potential of $2: 2$ rhythm is larger than that of the action potential of $1: 1$ rhythm. This longer recovery time also accounts for the fact that the delay seen at site $c$ is less for this action potential during period-2 rhythm than for the action potential of period-1 rhythm [compare upper rows of Figs. 1(B) and 1(C) and also action potentials in Figs. 3 and 4(A)].

In summary, as $\left[K^{+}\right]_{0}$ is raised from 13.39 to $13.40 \mathrm{mM}$ the sheet as a whole makes a transition from a global period-1 rhythm to a global period-2 rhythm with each element in the sheet responding with a period- 2 rhythm. Outside the ischemic core, propagating action potentials of two different morphologies can be identified on alternate beats. Within the ischemic core, one instead finds a maintained small-amplitude response on alternate beats. Further elevation of $\left[\mathrm{K}^{+}\right]_{0}$ in the ischemic zone to $13.45 \mathrm{mM}$ results in this response decaying electrotonically, producing an area of 2:1 block within the ischemic core [Figs. 1(C), 4].

\section{Higher-order rhythms}

The period-2 response with a 2:1 block in the ischemic core persists to $\left[K^{+}\right]_{0}=13.69 \mathrm{mM}$. Increasing $\left[K^{+}\right]_{0}$ by only $0.01 \mathrm{mM}$ to $13.70 \mathrm{mM}$ results in the $2: 1$ response in the core being abruptly replaced with a 4:1 response. Figure 6 shows the action potential waveforms and the corresponding maximum voltage maps for the four beats of the period-4 cycle. For three of the four beats of the cycle the action potential decrements to the point where it vanishes, leaving at most a barely discernable subthreshold response at location $c$ (the bottom three rows of Fig. 6) and a core of unexcited myocardium (maximum voltage maps in the rightmost column of Fig. 6).

Examination of the voltage waveform at locations between sites $a$ and $c$ reveals that there is a transformation ("spatial bifurcation', ) 38 of the local rhythm with increasing penetration into the ischemic zone. At sites $a$ and $b$, a 4:4 rhythm is seen (the first two columns of Fig. 6). As one moves deeper into the ischemic zone, the first action potential to drop out is the one in the second row of Fig. 6, thereby producing a local 4:3 rhythm. As one moves still deeper into the ischemic zone, the second action potential to drop out is the one in the last row, resulting in a local 4:2 response. The third and last to die out is the one in the third row, producing a $4: 1$ rhythm (e.g., site $c$ : third column of Fig. 6). This order of dropout is also reflected in the relative sizes of the areas of block for the respective beats (see voltage maximum maps of the ischemic area in the extreme-right column of Fig. 6). The sequence of rhythms observed locally as one traverses the 

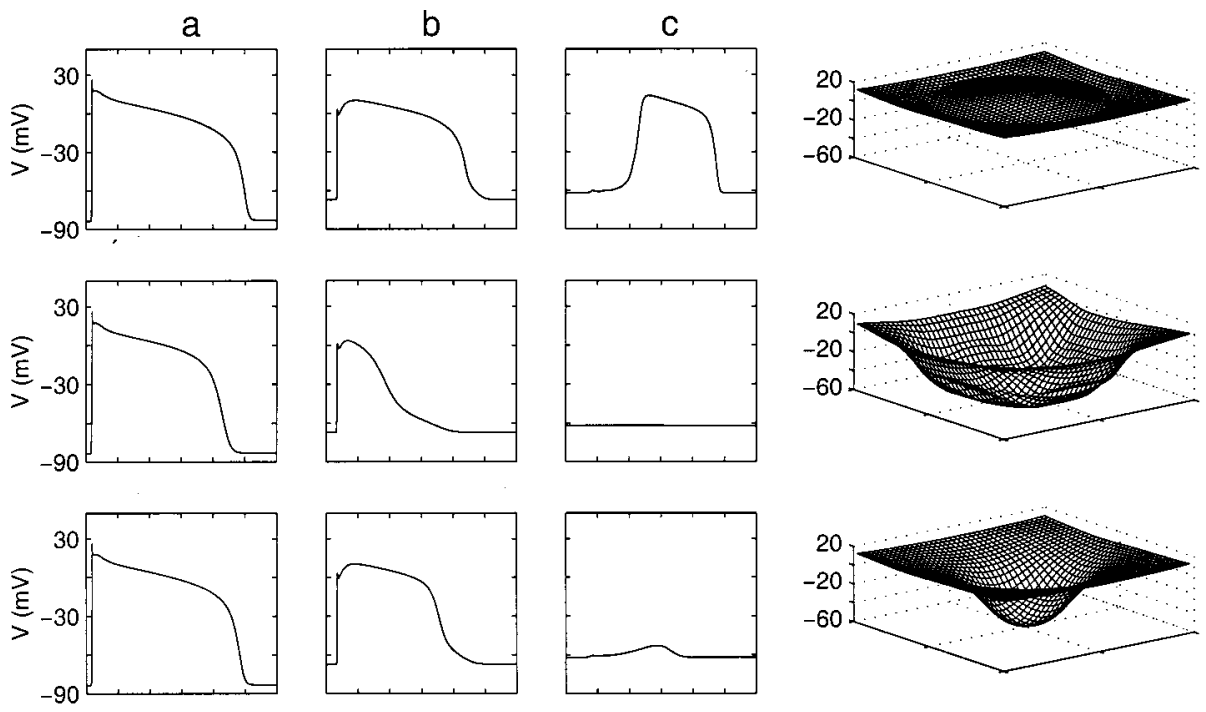

FIG. 6. Period-4 rhythm $\left(\left[\mathrm{K}^{+}\right]_{0}\right.$ $=13.7 \mathrm{mM}$ ). Transmembrane potential (at same three sites as in Fig. 1) and maximum voltage maps on the four beats of the period- 4 cycle. The beat shown in the first row is the conducted beat; the other three beats are blocked, each at a different point along the conduction pathway (see the text for a further description).
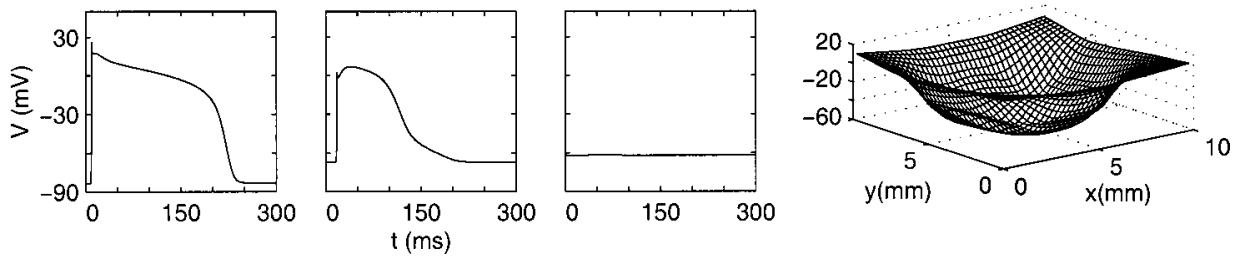

ischemic zone is thus $\{4: 4 \rightarrow 4: 3 \rightarrow 4: 2 \rightarrow 4: 1\}$. Bifurcations are not involved in this sequence; rather there is a change in a quantity corresponding to the winding number or rotation number in periodically stimulated limit-cycle oscillators. ${ }^{89}$ Again, the 4:4 rhythm might be mistakenly identified as 1:1 rhythm (or perhaps 2:2 rhythm) in an area sufficiently remote from the central area of $4: 1$ block.

The period-4 rhythm exists for $\left[K^{+}\right]_{0}=13.70-13.72$ $\mathrm{mM}$. A further increase in $\left[K^{+}\right]_{0}$ results in a transition to a period-6 rhythm, with 6:1 block in the central ischemic core, for $\left[K^{+}\right]_{0}=13.729-13.733 \mathrm{mM}$. As with the period-4 rhythm, there is a sequence of local rhythms seen as the ischemic area is traversed $\{6: 6 \rightarrow 6: 5 \rightarrow 6: 4 \rightarrow 6: 3 \rightarrow 6: 2 \rightarrow 6: 1\}$. Raising $\left[K^{+}\right]_{0}$ by $0.001 \mathrm{mM}$ to $13.734 \mathrm{mM}$ results in a period- 8 rhythm, with $8: 1$ block in the core (to identify this higher-order rhythm, it is necessary to inject $\sim 50$ stimuli). Finally, when $\left[K^{+}\right]_{0}$ is raised by another $0.001 \mathrm{mM}$ to $13.735 \mathrm{mM}$, there is a complete block in the ischemic core (i.e., no action potentials make it through), which agrees with the experimental finding that inexcitability occurs for $\left[K^{+}\right]_{0}>13.5 \mathrm{mM}^{66}$ At a value of $\left[K^{+}\right]_{0}$ where there is complete block, one can see a 1:0, 2:0, or 4:0 rhythm, ${ }^{90-92}$ depending on the precise value of $\left[K^{+}\right]_{0}$. In a 2:0 or 4:0 rhythm, the size of the subthreshold deflection is not invariant from beat to beat (2:0 rhythms have been recorded during ischemia-see, e.g., Fig. 5 of Ref. 6).

Our results over the range of $\left[K^{+}\right]_{0}$ that we have investigated are summarized in the first two columns of Table I. In brief, as $\left[\mathrm{K}^{+}\right]_{0}$ in the ischemic zone is raised from its normal value of $5.4 \mathrm{mM}$ up to $14 \mathrm{mM}$, one sees the following series of transitions: period-1 rhythm with orthograde transmission through entire ischemic zone $\rightarrow$ period-1 rhythm with retrograde transmission through a distal part of the ischemic zone $\rightarrow$ period-2 rhythm (2:2 response in the ischemic core followed by a $2: 1$ response) $\rightarrow$ period -4 rhythm $\rightarrow$ period- 6 rhythm $\rightarrow$ period- 8 rhythm $\rightarrow$ rhythms of complete block.

An obvious question is whether a different sequence of rhythms would be seen if the size of the sheet were to be changed. Due to the heavy computational requirements, we have investigated in detail only one other size of sheet: a 4 $\mathrm{cm} \times 4 \mathrm{~cm}$ sheet with a $2 \mathrm{~cm} \times 2 \mathrm{~cm}$ ischemic area. In that case we see essentially what we have reported above, with the sole exception being that the period- 6 rhythm is not seen; instead there is a transition from period- 4 to period- 8 rhythm as $\left[K^{+}\right]_{0}$ is raised from 13.460 to $13.465 \mathrm{mM}$.

\section{E. Role of retrograde activation wavefront}

As $\left[K^{+}\right]_{0}$ is raised, the first abnormality reported above was a slowing of anterograde conduction within the ischemic zone [Fig. 1(A)]. The next abnormality noticed was a departure from the normal activation sequence, with retrograde propagation and markedly delayed activation of a distal part of the ischemic zone [Fig. 1(B)]. At this level of $\left[K^{+}\right]_{0}$, there is a long delay $(\sim 50 \mathrm{~ms})$ from the peak of the first deflection in trace $c$ of Fig. 3 to the action potential upstroke [this can also be appreciated from the very long time that it takes the circle of unexcited tissue to become excited in the lower row of panels in Fig. 1(B)]. Note that by $t=50 \mathrm{~ms}$ [Fig. 1(B)] the two activation wavefronts traveling upwards and downwards in the normal area to the right of the ischemic area [Fig. 1(B): $t=40 \mathrm{~ms}$ ] have already merged; indeed, they have almost exited the sheet. However, at that time, the small-amplitude response at location $c$ is still present (Fig. 3: trace $c$ ). The very long delay in the region of delayed activation is presumably due to a combination of the 
very small amount of inward current available at this depolarized potential ${ }^{93}$ and geometrical effects due to the high curvature of the activation wavefront. ${ }^{94}$

The existence of three spatially-separated sources of excitation current (one anterograde, two retrograde) makes an analysis of what is happening in Fig. 1(B) very difficult. In particular, a natural question is whether this complicated spatial behavior is somehow involved in generating the bifurcation sequence reported above. We therefore simplified the situation by cutting away large areas at the top and bottom of the sheet, including the entire normal area above and below the ischemic zone, thus obliterating the source of the retrograde wavefront. This surgery leaves a thin strand of tissue 3 $\mathrm{cm}$ long and only $1.25 \mathrm{~mm}$ wide, with normal tissue at its left- and right-hand ends, and ischemic tissue in its middle.

As $\left[K^{+}\right]_{0}$ is raised in the strand, one again has a $1: 1$ rhythm for $\left[\mathrm{K}^{+}\right]_{0}$ sufficiently low. Unlike the case with the sheet [Fig. 1(B)], during 1:1 rhythm there is now always at most one activation wavefront present in the strand, making its way from left to right (again with decremental conduction as it enters the proximal border zone and incremental conduction as it leaves the ischemic zone). There is once again an abrupt transition to period-2 rhythm as $\left[\mathrm{K}^{+}\right]_{0}$ is raised by the almost infinitesimal amount of $1 \mu \mathrm{M}$ from 13.104 to $13.105 \mathrm{mM}$. During this period-2 rhythm, there is a $2: 2$ response consisting of alternating action potentials in the lefthand area of normal tissue. The degree of alternation increases with increasing penetration into the ischemic zone, until, within the ischemic core, the smaller of the two action potentials eventually converts into the maintained smallamplitude response, as in the sheet [the top panel of Fig. 5(B)]. However, unlike the case in the sheet, there is now incremental conduction out of the ischemic core, with the maintained small-amplitude response gradually turning into a full-fledged action potential. This results in the appearance of a second disjoint area of alternating action potentials just distal to the ischemic core, with the degree of alternation gradually decreasing as the ischemic zone is exited. This $2: 2$ exit response is not seen in the sheet, presumably because the incremental conduction in the anterograde direction does not have time to develop before the enveloping retrograde invasion occurs.

When $\left[K^{+}\right]_{0}$ is raised to $13.20 \mathrm{mM}$ in the strand, the maintained small-amplitude response within the proximal part of the ischemic core gradually turns into a decremental subthreshold response with increasing penetration into the ischemic core, so that one finally ends up with a 2:1 response within the distal end of the ischemic area. Since incremental conduction out of the ischemic core now vanishes, a 2:1 response is also found within the right-hand normal area distal to the ischemic zone. A further increase in $\left[\mathrm{K}^{+}\right]_{0}$ results in the period-2 rhythm being replaced successively with period-4, period-6, period-8, and complete-block rhythms, as in the sheet, but with each particular range of rhythms occurring at a slightly lower $\left[\mathrm{K}^{+}\right]_{0}$ than in the sheet (Table I). We believe that this difference occurs because the retrodradely invading wavefront and the two wavefronts moving in the surrounding normal area inject extra stimulation current into the ischemic zone, thus postponing the onset of block in the sheet with respect to the strand. The fact that we see the same sequence of periodicities over virtually the same ranges of $\left[\mathrm{K}^{+}\right]_{0}$ in the sheet and in the strand leads us to conclude that retrograde conduction is not absolutely crucial in generating the particular periodicities seen in the sheet. A similar correspondence holds in the computations made in a $4 \mathrm{~cm}$ long strand (with a $2 \mathrm{~cm}$ long ischemic area) cut out of the $4 \mathrm{~cm} \times 4 \mathrm{~cm}$ sheet: i.e., period-1, 2, 4, and 8 rhythms followed by complete block seen in both sheet and strand, with the transition from period-4 to period- 8 rhythm occurring as $\left[\mathrm{K}^{+}\right]_{0}$ is raised from 13.412 to $13.416 \mathrm{mM}$. All of the results in the strand given above were taken from the asymptotic behavior in runs in which 64 stimuli were injected.

\section{F. Reentrant rhythms}

Based on the fact that alternans often precedes arrhythmias (see the references in the Introduction), we were eagerly anticipating the induction of reentrant arrhythmias at the point that $\left[K^{+}\right]_{0}$ was raised to a level somewhat higher than that at which alternans first emerged. This expectation was reinforced when retrograde activation ("concealed reentry") and arcs of conduction block, which are often harbingers of reentry, were seen within the ischemic zone during alternans rhythm in Fig. 1(C) [and indeed even before that point, during 1:1 rhythm with retrograde activation, Fig. 1(B)]. In addition, the arc of block effectively breaks the activation wavefront into two separate fragments, creating two free-ends resembling incipient spiral-wave tips [e.g., Fig. 1(B): $t=40 \mathrm{~ms}$ ]. Should the right-hand part of the ischemic zone be out of its refractory period at the point that these two nascent spiral tips arrive at its distal margin, one can imagine that they would merge and perhaps successfully propagate retrogradely through the ischemic zone all the way to its proximal border. Upon emerging into the normal area to the left of the ischemic zone, two wavefronts would then circulate around the ischemic area traveling in the anterograde direction within the normal areas above and below the ischemic zone, thus initiating a figure-of-eight reentrant rhythm. The above scenario is based on results recently obtained in a modeling study on the effect of fast pacing when the LR model is modified to produce an APD that is much shorter than that used in the present study (see Fig. 7 of Ref. 70). However, we found that the ischemic zone did not come out of refractoriness soon enough to allow this to occur in either the $3 \mathrm{~cm} \times 3 \mathrm{~cm}$ sheet or the $4 \mathrm{~cm} \times 4 \mathrm{~cm}$ sheet.

\section{DISCUSSION}

\section{A. Primary and secondary alternans}

Beat-to-beat alternation of action potential morphology can be seen in situations in which spatial factors are clearly not involved: e.g., a single ventricular cell ${ }^{87,88,95,96}$ or an ionic model of isopotential ventricular membrane ${ }^{87,97,98} \mathrm{In}$ these cases, the excitation current injected into the cell is maintained constant from beat to beat by artificial external means, and there are no adjacent cells to act as sources or sinks of coupling current. This form of alternans, which is an inherent property of isopotential or space-clamped mem- 
brane, has been referred to as "primary" 99 or "intrinsic" 100 alternans. Primary alternans is the normal response of multicellular ventricular muscle to fast pacing, ${ }^{35,45,101-106}$ and there is considerable evidence that the main requirement for obtaining primary alternans is a sufficiently steep APD restitution curve at short recovery intervals. ${ }^{43,46,104,107-109}$ In contrast, the alternans of action potential morphology seen in our simulations can be considered to be "secondary" or "extrinsic," since there is a concurrent beat-to-beat alternation in the flow of intercellular current into and out of the site at which alternans in the action potential morphology is seen (e.g., at location $b$ in Fig. 4 or Fig. 5).

Over a hundred years ago Gaskell suggested that mechanical alternans in multicellular tissue could be accounted for by a situation in which there is a subpopulation of cells responding in an abnormal 2:1 fashion, while the rest of the cells display their usual 1:1 rhythm. ${ }^{110}$ Gaskell's original scenario of 1:1 and 2:1 subpopulations is played out in a cellular automaton type of model incorporating spatial dispersion of refractory period where there are discrete elements with no electrotonus. ${ }^{111}$ In one of the two different period-2 rhythms that we see in the sheet, there are two subpopulations of cells, one with a 2:2 response, the other with a 2:1 response [Figs. 1(C), 4]. This form of alternans is thus a variant of that proposed by Gaskell, with his 1:1 response being replaced, because of electrotonic coupling, by our $2: 2$ response (which asymptotically becomes a 1:1 response infinitely far from the center of the ischemic area). The other form of period-2 rhythm that we see in the sheet is maintained over an extremely narrow range of $\left[\mathrm{K}^{+}\right]_{0}$ and consists of an alternation between a full-sized action potential and the maintained small-amplitude response (Fig. 5). We are not aware of any prior descriptions of alternans involving this small-amplitude response, which occurs when there is just barely enough inward current available to support a propagating response.

The prolongation of the refractory period and flattening of the APD restitution curve known to be induced by ischemia ${ }^{29,79,112}$ would mitigate against the development of primary alternans (an acidosis-induced flattening of the mechanical restitution curve has also been described). ${ }^{95}$ However, work on single rabbit ventricular cells, ${ }^{83}$ aggregates of embryonic chick ventricular cells, ${ }^{47}$ and the isopotential LR model $^{83}$ shows that the transition from 1:1 rhythm to primary alternans seen at high stimulus amplitude is replaced by a direct transition to $2: 1$ rhythm when the stimulus amplitude is reduced. This occurs essentially because the refractory period is made longer, so destroying the steeper part of the APD restitution curve that is essential to the development of primary alternans. ${ }^{83}$ It is therefore possible that during ischemia there will be a direct transition to $2: 1$ block in a subpopulation of cells that would then induce secondary alternans in neighboring cells. In contrast to the above argument, it has also been suggested that ischemia changes the shape of the APD restitution curve in such a fashion so as to promote primary alternans during ischemia (see Fig. 5 of Ref. 112).

The electrophysiological mechanisms underlying alternans in ischemic ventricular muscle are yet to be fully deter- mined. In particular, it is not clear whether there is an obligatory region of 2:1 block somewhere in the ventricles during ischemic alternans, as in Fig. 1(C) and Fig. 4, since there are several reports on ischemic alternans that make no mention of 2:1 block. However, in virtually all of those cases, either a measurement is made at only one site in the ventricle or the summated activity of ventricular muscle is recorded in the electrogram or electrocardiogram. There is thus no guarantee in those instances that there is not a site of 2:1 block somewhere else in the ventricle. Indeed, unless one makes transmural recordings at multiple sites, one cannot rule out the existence of a region of 2:1 block. Nevertheless, in one report in which activation mapping was made on a fine spatial scale during ischemic alternans, there was indeed a 2:1 response in the center of the ischemic region, with the largest degree of alternation in the unipolar electrogram being seen only $3 \mathrm{~mm}$ away, along the border of the ischemic zone (Fig. 5 of Ref. 27). There are also several other reports showing a simultaneous presence of 2:2 and 2:1 rhythms at two close sites (e.g., Fig. 9 of Ref. 18, Fig. 1 of Ref. 21, Fig. 5 of Ref. 27, and Fig. 3 of Ref. 30).

All of the simulations reported on above have been conducted at one fixed BCL (400 ms). At that BCL, $\left[\mathrm{K}^{+}\right]_{0}$ must be raised considerably above the normal level of $5.4 \mathrm{mM}$ to obtain alternans. However, we expect that primary alternans would occur in our model at normal $\left[\mathrm{K}^{+}\right]_{0}$ should a BCL shorter than $400 \mathrm{~ms}$ be used:-in fact, we then expect it to occur in the normal area, since, as mentioned above, primary alternans is the normal response of healthy multicellular ventricular muscle to fast pacing, and sustained alternans can be seen at a shorter BCL in the space-clamped version of our modified version of the LR model [the alternans described in the original LR space-clamped model (Fig. 19 of Ref. 76) is a transient]. In a recent modeling study using a differently modified version of the LR model, it was found that fast pacing could induce primary alternans in a two-dimensional sheet. ${ }^{113}$ In another recent study in which fast pacing was used to induce alternans and arrhythmias in the non-ischemic ventricle, there was no mention made of $2: 1$ block being seen at any of the 128 epicardial recording sites, which suggests the presence of primary alternans. ${ }^{105}$

There is also evidence that an intrinsic primary alternation of internal $\mathrm{Ca}^{++}$levels, particularly during ischemic conditions, can induce alternans in membrane potential, by, e.g., involving $\mathrm{Ca}^{++}$-activated currents and the $\mathrm{Na}^{+}-\mathrm{Ca}^{++}$ exchanger. ${ }^{29,95,114-118}$ This view is bolstered by the fact that adding agents that decrease internal $\mathrm{Ca}^{++}$levels (e.g., ryanodine, ${ }^{96,103,115,119}$ caffeine, ${ }^{96,103,115,116,119}$ and calciumchannel blockers ${ }^{102,120}$ ) abolishes or markedly reduces the alternans produced by pacing nonischemic tissue at a fast rate. Ischemic alternans and the incidence of ventricular arrhythmias are also reduced or abolished by administration of a $\mathrm{Ca}^{++}$-channel blocker. ${ }^{8,28,34,114,121-123}$ However, since $\mathrm{Ca}^{++}$-channel blockers reduce the slope of the APD restitution curve,${ }^{106}$ they may be acting to abolish or reduce alternans through a mechanism involving APD restitution. There is also evidence supporting a role for intracellular $\mathrm{Ca}^{++}$-cycling in alternans and arrhythmia in an animal model for the long-QT syndrome. ${ }^{118}$ To investigate electrical 
alternans induced by an intrinsic alternation in internal $\mathrm{Ca}^{++}$ dynamics would necessitate using a "second-generation" model incorporating Ca-activated currents and the $\mathrm{Na}^{+}-\mathrm{Ca}^{++}$exchange current. However, before this can be done, problems associated with degeneracy ${ }^{74,75}$ and long drifts $^{70,72,73}$ in this class of model will have to be worked out.

\section{B. Bifurcation from period-1 to period-2 rhythm}

There have been several different bifurcation routes implicated in different instances of the transition from period-1 to period-2 rhythm in cardiac tissue. The transition from 1:1 rhythm to primary alternans that occurs as BCL is decreased

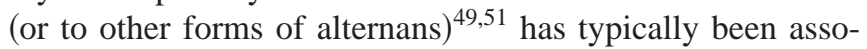
ciated with a supercritical period-doubling bifurcation on a one-dimensional map in both experimental and modeling work. $43,46,58,89,97,104,107-109,124,125$ When the period-doubling bifurcation is supercritical, there is a gradual growth in the alternans amplitude from zero as the BCL (the bifurcation parameter) is decreased. However, analysis of onedimensional maps obtained from consideration of the APD restitution curve suggests that alternans can also arise at a nonzero amplitude as the result of a saddle-node bifurcation of period-doubled orbits. ${ }^{108,124}$ In one of these two reports, the unstable period-doubled branch produced in this bifurcation terminates in a subcritical period-doubling bifurcation involving the period-1 orbit [Fig. 5(A) of Ref. 124]. In the other case, this unstable branch terminates in another saddlenode bifurcation of period-doubled orbits, the stable member of which terminates in a supercritical period-doubling bifurcation involving the period-1 orbit [Fig. 8(B) of Ref. 108]. In addition, a direct transition from 1:1 to $2: 1$ rhythm has been seen at an intermediate stimulus amplitude in single rabbit ventricular cells and the original LR space-clamped model. ${ }^{83}$ This direct $\{1: 1 \rightarrow 2: 1\}$ transition can be accounted for by a two-branched discontinuous map, with a stable period-1 orbit on the left-hand branch of the map corresponding to $1: 1$ rhythm, and a second stable period-1 orbit on the right-hand branch corresponding to 2:1 rhythm (see Fig. 9 of Ref. 83). The discontinuity in the map arises because of the essentially all-or-none nature of excitation.

As $\left[K^{+}\right]_{0}$ is raised in our study, immediately upon the transition from period-1 to period- 2 rhythm, one sees a beatto-beat alternation within the ischemic core between an action potential and the maintained small-amplitude response, both in the strand and in the sheet (Fig. 5). One possibility is that this is a smooth transition generated by a supercritical period-doubling bifurcation, which, just beyond the bifurcation point, would generate a rhythm consisting of two alternating action potentials of arbitrarily close morphology within the ischemic core. However, we have not found any evidence for such a smooth transition using an increment in $\left[\mathrm{K}^{+}\right]_{0}$ as small as $0.001 \mathrm{mM}$ in the strand. Thus, the more likely possibility is that the transition involves a subcritical period-doubling bifurcation, or some sort of effectively discontinuous transition, as in the direct $\{1: 1 \rightarrow 2: 1\}$ transition mentioned just above.

\section{Bistability}

In each of our runs at a given $\left[\mathrm{K}^{+}\right]_{0}$, stimulation was started from one set of initial conditions approximating steady-state rest conditions at that $\left[\mathrm{K}^{+}\right]_{0}$. There is now much evidence in a variety of experimental and modeling situations that $2: 1$ rhythm can be bistable with either $1: 1$ or 2:2 rhythm. ${ }^{43,47,83,87,108,125-127}$ In addition, a 2:2 rhythm can sometimes be bistable with a $1: 1$ rhythm $^{97,124}$ or with a second 2:2 rhythm. ${ }^{107,108,124}$ Thus, an investigation should be carried out to establish whether bistability exists here. The probable existence of bistability complicates carrying out a definitive characterization of the bifurcation structure, imposing an extremely heavy computational load, which might even be prohibitively large at the present time in the case of the sheet. Nevertheless, such a search should be made, even if only partial, since it has been recently suggested ${ }^{25}$ that such bistability might be involved in "triggered alternans, ${ }^{, 5,15,29}$ and in the conversion of discordant into concordant alternans. ${ }^{25,96}$

\section{Higher-order period-doubled rhythms}

There are very few reports of higher-order perioddoubled rhythms in ventricular muscle in conditions predisposing to arrhythmias. In particular, we know of only one case-involving a toad ventricle driven at a progressively faster rate-in which arrhythmias occurred following a period-doubling cascade culminating in chaos (the sequence of rhythms seen was $\{1: 1 \rightarrow 2: 2 \rightarrow 4: 4 \rightarrow$ chaos $\rightarrow$ arrhythmias $\}$ ). ${ }^{45}$ There are however isolated reports of period-4 rhythms (with traces consistent with periodquadrupled orbits, as in Fig. 5) in ventricular muscle in two other conditions predisposing to arrhythmias: ischemia [Fig. 2(C) of Ref. 29] and hypothermia (Fig. 4.17 of Ref. 100). The relative rarity of these higher-order rhythms is almost certainly due to the fact that the range of the parameter over which they can be maintained is so very small. For example, in our $3 \mathrm{~cm} \times 3 \mathrm{~cm}$ sheet, the period- 4 rhythm is maintained over a range of $\left[\mathrm{K}^{+}\right]_{0}$ of somewhere between 0.02 and 0.04 $\mathrm{mM}$ (Table I), a range so narrow that it virtually guarantees that the rhythm would not be seen in the corresponding experiment (the same conclusion also holds for the period-6 and period-8 rhythms, which exist over even narrower ranges of $\left.\left[\mathrm{K}^{+}\right]_{0}\right)$. Thus, in the real world of the clinic or the laboratory, one would expect to see a bifurcation gap, with a transition from 1:1 rhythm (with delayed activation) to alternans and then to complete block as ischemia progresses. This is exactly what has been described several times during ischemia. ${ }^{6,16,18,19}$ In our simulations we have not seen "tripling," in which there is a period-3 rhythm, probably arising from 3:2 Wenckebach block or 3:1 high-grade block. ${ }^{10,21,26}$

\section{E. Period-doubling route to chaos?}

The existence of period- 2 and period-4 rhythms in ischemia, together with the experimental ${ }^{42,45-47,88,109}$ and modeling ${ }^{97,108,128,129}$ evidence for the period-doubling route to chaotic dynamics in cardiac tissue, leads very naturally to consideration of the possibility that the close temporal asso- 
ciation of ventricular fibrillation with alternans might be an indication that ventricular fibrillation is a chaotic phenomenon somehow arising out of a cascade of period-doubling bifurcations. $9,32,37,38,52,53,111$ Our results in the larger sheet ( $4 \mathrm{~cm} \times 4 \mathrm{~cm}$, with a $2 \mathrm{~cm} \times 2 \mathrm{~cm}$ ischemic area) and the corresponding strand might be taken as providing some support for this hypothesis, in that we see the sequence fperiod$1 \rightarrow$ period $-2 \rightarrow$ period $-4 \rightarrow$ period $-8 \rightarrow \cdots\}$. However, as for the transition from period-1 to period- 2 rhythm discussed above, the transitions involving the higher-order rhythms are so very abrupt that we have not been able to document the existence of supercritical period-doubling bifurcations. In addition, in the smaller sheet $(3 \mathrm{~cm} \times 3 \mathrm{~cm}$, with a $1 \mathrm{~cm} \times 1$ $\mathrm{cm}$ ischemic area) and the corresponding strand, the sequence departs from the classic period-doubling cascade, since a period- 6 rhythm is interposed between the period- 4 and period-8 rhythms. One theoretical possibility that would account for this difference is that the period- 6 rhythm might be bistable with either the period- 4 or the period- 8 rhythm (or both), and so would be seen in some computations, but not others. Another possible reason for the absence of this rhythm in the larger sheet and strand is that we might have missed it in our computations (the period- 4 rhythm goes to period- 8 rhythm with a change in $\left[K^{+}\right]_{0}$ of $0.005 \mathrm{mM}$ in the sheet and $0.004 \mathrm{mM}$ in the strand). As usual, there is of course no guarantee that numerical integration with finite precision will necessarily find all stable periodic orbits. A very large amount of computation will be required to determine the full two-parameter $\left(\left[K^{+}\right]_{0}\right.$, system size $)$ bifurcation diagram, especially in the sheet, and even moreso should bistability exist. We anticipate that, as in prior studies, ${ }^{43,46,108,109}$ determination of the APD restitution curve and iteration of a finite-difference equation or map derived from it will be essential in order to make any headway in understanding the global organization of the bifurcation diagram.

While we have as yet no firm evidence of chaotic behavior in our simulations, there are several other modeling studies on spatially-extended electrophysiological systems showing apparently chaotic behavior, ${ }^{56-58,61,90,92,108,129-131}$ sometimes as the result of a period-doubling cascade. It is also quite possible that the spiral- or scroll-wave breakup seen in modeling work ${ }^{56-61,113,132}$ might be an example of " "extensive chaos,",133 perhaps corresponding to ventricular fibrillation.

\section{F. Alternans and reentrant arrhythmias}

" Clinically, the physician must recognize that electrical alternans in angina pectoris is a sign preceding sudden death.' Kataoka and Yoshimura. ${ }^{5}$

While we have seen several phenomena in our simulations corresponding to the prodroma presaging the imminent onset of reentrant arrhythmias in experiment (e.g., slowresponse action potentials, increased conduction delay, twocomponent upstrokes, retrograde propagation, concealed reentry, arcs of block, nascent spiral tips, alternans), these have not been followed by sustained reentrant rhythms. The major factor preventing reentry in our study seems to be that the ischemic zone is still refractory when retrograde invasion starts. We thus believe that reentry might occur should the size of the ischemic zone be made larger or additional interventions (e.g., acidosis and hypoxia) that are known to reduce APD and conduction velocity further within the ischemic zone included in the model. Indeed, while regional perfusion with a high-K solution mimics many of the phenomena seen in ischemia, including a phase of alternans preceding arrhythmia onset, ${ }^{69}$ regional perfusion ${ }^{68}$ or superfusion $^{134}$ with a high-K, acidotic, hypoxic, glucose-free solution more closely mimics the electrophysiologic changes seen during ischemia. In addition, acidosis can induce primary alternans in single ventricular cells paced at $\mathrm{BCL}=500$ $\mathrm{ms},{ }^{95}$ superfusion of ventricular muscle with either a hypoxic $^{135,136}$ or acidotic ${ }^{136}$ solution can induce alternans, and administration of sodium bicarbonate to correct acidosis can abolish ischemic alternans. ${ }^{5}$ Further work attempting to induce reentry should thus use a more complicated model incorporating effects due to acidosis, hypoxia, and metabolic inhibition. ${ }^{137-139} \mathrm{We}$ anticipate that the onset of reentry will only occur following the induction of a 2:1 response in the ischemic core, because not only does the wavefront then have two broken ends [Fig. 1(C)] that could potentially curl up, forming the tips of two spiral waves, but the anterograde block within the ischemic zone would then result in a recovery time long enough to permit subsequent retrograde propagation through that area. ${ }^{70}$

Another possible reason for the absence of arrhythmias in our study is that we have used a very simple form of large-scale inhomogeneity: a centrally-placed homogeneous square. However, the ischemic zone in reality is inhomogeneous, having a graded border zone with irregular boundaries and small-scale inhomogeneities in $\left[\mathrm{K}^{+}\right]_{0}$ that lead to significant dispersion of refractoriness, ${ }^{65-67}$ which is the classic substrate for the formation of reentrant arrhythmias. Future modeling work should thus also investigate the extent to which a spatially-extended intrinsic border zone and smallerscale inhomogeneities are arrhythmogenic.

Our inability to observe reentry following alternans might nevertheless be consistent with experimental and clinical observations that arrhythmias do not invariably start up following alternans. In one experimental study, $72 \%$ of the occlusion episodes that did not eventually result in fibrillation nevertheless demonstrated alternans, and 33\% of these episodes showed evidence of conduction block. ${ }^{21}$ Alternans that is not detectable to the naked eye can be found in some patients at rest or during exercise in the absence of spontaneous arrhythmias. ${ }^{140}$ The most obvious explanation for these results is that the ischemic insult was not profound enough to proceed beyond the stage of alternans (perhaps this is what is effectively happening in our simulations in which alternans is not followed by reentrant arrhythmias). In some clinical episodes of ischemia in which alternans occurs, but arrhythmias do not arise, prolongation of the duration of the ischemic episode can then bring about arrhythmias. ${ }^{8}$ In experiments in which alternans does not culminate in arrhythmias, increasing the rate of pacing, which results in an increase in alternans amplitude, ${ }^{12,123}$ can then provoke arrhythmias. ${ }^{18,21}$ This might be the result of an increase in 
the amount of activation delay and in an extension in the size of the area of 2:1 block, or perhaps even the induction of primary alternans at the shorter BCL. In addition, there are cases on record in which the alternans appears and disappears, and arrhythmias then start up. ${ }^{141}$ This could conceivably be due to an increased effect of ischemia leading to the alternans proceeding to the stage of complete block within the ischemic zone, which would then predispose to reentry.

Just as alternans does not necessarily proceed on to arrhythmias, alternans is not invariably seen before arrhythmias occur. There are several possible explanations for this finding. First, the period of alternans can be quite brief, and so it might be missed. ${ }^{6}$ Second, the regions of alternans and $2: 1$ block might be too small in size to produce obvious alternation in the surface electrocardiogram. In an experimental study using voltage-sensitive dyes in the nonischemic heart, rapid pacing produced primary alternans at all sites, but alternans was not visible in the electrocardiogram until the BCL was decreased sufficiently (Fig. 4 of Ref. 105). In one clinical study on ischemia, moving the monophasic action potential electrode a distance of $1 \mathrm{~cm}$ away from the ischemic area resulted in the disappearance of alternans ${ }^{10}$ (see also Refs. 27 and 30). These observations, taken together with our simulations showing that global 1:1 synchronization is lost when there is a sudden switch to period-2 rhythm within a relatively small area, support the use of sensitive signal detection techniques such as the power spectrum, ${ }^{9,13,37}$ complex demodulation, ${ }^{12,32-34}$ and Karhunen-Loève decomposition ${ }^{142}$ to detect occult alternans. A third explanation for the absence of alternans before arrhythmias are seen is simply that alternans does not exist before every ischemic arrhythmia occurs. In some instances of acute ischemia, ${ }^{18,26,81}$ as well as in many instances during the later stages of myocardial infarction, ${ }^{82,143}$ arrhythmias are preceded by Wenckebach or 3:1 block, and not alternans. Again, this finding might be consistent with the results from studies on single ventricular cells, ${ }^{84-86}$ isopotential aggregates of embryonic ventricular cells, ${ }^{47}$ and the unmodified LR model, ${ }^{86}$ where Wenckebach rhythms are seen only when the stimulus amplitude is very low.

While we have focused above entirely on ischemia, there is also evidence for alternans being associated with malignant ventricular arrhythmias in several other settings: e.g., hypothermia, ${ }^{37}$ long-QT syndrome (see the references in Refs. 1 and 2), electrolyte imbalance (see the references in Ref. 1), administration of anti-arrhythmic drugs, ${ }^{144}$ and fast pacing. ${ }^{45,105}$ The exact nature of any causal connection between alternans and malignant ventricular arrhythmiaspresumably spiral-wave reentrant in origin-in acute myocardial ischemia and these other situations remains to be clarified.

\section{ACKNOWLEDGMENTS}

We thank Jesús Gutiérrez for help in running programs and Ali Yehia for much general help with computers. We also thank the Medical Research Council of Canada (MG) and Dirección General de Asuntos del Personal Académico-
UNAM (HA, HG) for financial support of this work, as well as Dirección General de Servicios de Cómputo-UNAM (HA) for supercomputer time.

${ }^{1}$ B. Surawicz and C. Fisch, "Cardiac alternans: diverse mechanisms and clinical manifestations,' J. Am. Coll. Cardiol. 20, 483-499 (1992).

${ }^{2}$ M. A. Murda'h, W. J. McKenna, and A. J. Camm, "'Repolarization alternans: techniques, mechanisms, and cardiac vulnerability,', PACE 20, 2641-2657 (1997).

${ }^{3}$ R. R. Williams, G. S. Wagner, and R. H. Peter, "ST-segment alternans in Prinzmetal's angina. A report of two cases,' Ann. Int. Med. 81, 51-54 (1974).

${ }^{4}$ M. J. Kleinfeld and J. J. Rozanski, "Alternans of the ST segment in Prinzmetal's angina,' Circulation 55, 574-577 (1977).

${ }^{5} \mathrm{~K}$. Kataoka and R. Yoshimura, "Clinical and experimental studies on electrical alternans of ST-segment and T-wave in variant form of angina pectoris,' Resp. Circ. 27, 767-776 (1979) (in Japanese).

${ }^{6}$ J. Cinca, M. J. Janse, H. Moréna, J. Candell, V. Valle, and D. Durrer, "Mechanism and time course of the early electrical changes during acute coronary artery occlusion,', Chest 77, 499-505 (1980).

${ }^{7}$ G. Demidowich, R. Werres, D. Rothfeld, and J. Becker, "Electrical alternans of the ST segment in non-Prinzmetal's angina,' PACE 3, 733-736 (1980).

${ }^{8}$ J. A. Salerno et al., "Ventricular arrhythmias during acute myocardial ischaemia in man. The role and significance of R-ST-T alternans and the prevention of ischaemic sudden death by medical treatment,' Eur. Heart J. 7, 63-75 (1968).

${ }^{9}$ J. M. Smith, E. A. Clancy, C. R. Valeri, J. N. Ruskin, and R. J. Cohen, "Electrical alternans and cardiac electrical instability,', Circulation 77, 110-121 (1988).

${ }^{10}$ P. M. I. Sutton, P. Taggart, M. Lab, M. E. Runnalls, W. O’Brien, and T. Treasure, "Alternans of epicardial repolarization as a localized phenomenon in man,' Eur. Heart J. 12, 70-78 (1991).

${ }^{11}$ E. A. Raeder, D. S. Rosenbaum, R. Bhasin, and R. J. Cohen, "Alternating morphology of the QRST complex preceding sudden death,' N. Engl. J. Med. 326, 271-272 (1992).

${ }^{12}$ B. D. Nearing, S. N. Oesterle, and R. L. Verrier, "Quantification of ischaemia induced vulnerability by precordial $\mathrm{T}$ wave alternans analysis in dog and human,' Cardiovasc. Res. 28, 1440-1449 (1994).

${ }^{13}$ D. S. Rosenbaum, L. E. Jackson, J. M. Smith, H. Garan, J. N. Ruskin, and R. J. Cohen, "Electrical alternans and vulnerability to ventricular arrhythmias,' N. Engl. J. Med. 330, 235-241 (1994).

${ }^{14}$ V. Chini, "Ulteriore contributo alla conoscenza dell'alternanza. (Ricerche sperimentali sul cuore di coniglio),' Cuore Circolazione 12, 494-506 (1927).

${ }^{15}$ H. K. Hellerstein and I. M. Liebow, "Electrical alternation in experimental coronary artery occlusion,' Am. J. Physiol. 160, 366-374 (1950).

${ }^{16}$ L. Palagi, S. Sonnino, M. Puletti, G. F. Jacobellis, and L. Baschieri, "Indagini su l'importanza del ritardo di attivazione del miocardio $\ll$ ischemico $\gg$ nella genesi della corrente di lesione,' Cuore Circolazione 50, 248-260 (1966)

${ }^{17}$ H. A. Roselle, R. S. Crampton, and R. B. Case, "Alternans of the depressed S-T segment during coronary insufficiency,', Am. J. Cardiol. 18, 200-207 (1966).

${ }^{18}$ E. Downar, M. J. Janse, and D. Durrer, "The effect of acute coronary artery occlusion on subepicardial transmembrane potentials in the intact porcine heart,' Circulation 56, 217-224 (1977).

${ }^{19}$ A. G. Kléber, M. J. Janse, and F. J. L. van Capelle, "Mechanism and time course of S-T and T-Q segment changes during acute regional myocardial ischemia in the pig heart determined by extracellular and intracellular recordings,"' Circulation 42, 603-613 (1978).

${ }^{20}$ M. Nakashima, H. Hashimoto, M. Kanamaru, T. Nagaya, M. Hashizume, and H. Oishi, "Experimental studies and clinical report on the electrical alternans of ST segment during myocardial ischemia,' Jpn. Heart J. 19, 396-408 (1978)

${ }^{21}$ D. C. Russell, H. J. Smith, and M. F. Oliver, "Transmembrane potential changes and ventricular fibrillation during repetitive myocardial ischaemia in the dog," Br. Heart J. 42, 88-96 (1979).

${ }^{22}$ M. J. Janse, H. Moréna, J. Cinca, J. W. T. Fiolet, W. J. Krieger, and D. Durrer, "Electrophysiological, metabolic and morphologic aspects of acute myocardial ischemia in the isolated porcine heart. Characterization of the «border zone»,' J. Physiol. (Paris) 76, 785-790 (1980).

${ }^{23}$ T. Akiyama, "Intracellular recording of in situ ventricular cells during 
ventricular fibrillation," Am. J. Physiol. 240, H465-H471 (1981).

${ }^{24}$ J. Cinca, J. Figueras, G. Senador, E. García-Moreno, A. Salas, and J. Rius, "Transmural DC electrograms after coronary artery occlusion and latex embolization in pigs," Am. J. Physiol. 246, H475-H482 (1984).

${ }^{25} \mathrm{H}$. Hashimoto, K. Suzuki, and M. Nakashima, "Effects of the ventricular premature beat on the alternation of the repolarization phase in ischemic myocardium during acute coronary occlusion in dogs," J. Electrocardiol. 17, 229-238 (1984).

${ }^{26}$ H. Hashimoto, M. Asano, and M. Nakashima, "Potentiating effects of a ventricular premature beat on the alternation of the ST-T complex of epicardial electrograms and the incidence of ventricular arrhythmias during acute coronary occlusion in dogs," J. Electrocardiol. 17, 289-302 (1984).

${ }^{27}$ D. L. Carson, R. Cardinal, P. Savard, and M. Vermeulen, "Characterisation of unipolar waveform alternation in acutely ischaemic porcine myocardium," Cardiovasc. Res. 20, 521-527 (1986).

${ }^{28}$ H. Hashimoto, M. Asano, and M. Nakashima, "Alternation in refractoriness and in conduction delay in the ischemic myocardium associated with the alternation in the ST-T complex during acute coronary occlusion in anesthetized dogs," J. Electrocardiol. 19, 77-84 (1986).

${ }^{29}$ S. G. Dilly and M. J. Lab, "Electrophysiological alternans and restitution during acute regional ischaemia in myocardium of anaesthetized pig," J. Physiol. (London) 402, 315-333 (1988).

${ }^{30}$ S. Abe, Y. Nagamoto, Y. Fukuchi, T. Hayakawa, and A. Kuroiwa, "Relationship of alternans of monophasic action potential and conduction delay inside the ischemic border zone to serious ventricular arrhythmia during acute myocardial ischemia in dogs," Am. Heart J. 117, 1223-1233 (1989).

${ }^{31}$ T. Konta, K. Ikeda, M. Yamaki, K. Nakamura, K. Honma, I. Kubota, and S. Yasui, "Significance of discordant ST alternans in ventricular fibrillation," Circulation 82, 2185-2189 (1990).

${ }^{32}$ B. D. Nearing, A. H. Huang, and R. L. Verrier, "Dynamic tracking of cardiac vulnerability by complex demodulation of the T wave," Science 252, 437-440 (1991).

${ }^{33}$ R. L. Verrier and B. D. Nearing, "Electrophysiologic basis for T wave alternans as an index of vulnerability to ventricular fibrillation," J. Cardiovasc. Electrophysiol. 5, 445-461 (1994).

${ }^{34}$ B. D. Nearing, J. J. Hutter, and R. L. Verrier, "Potent antifibrillatory effect of combined blockade of calcium channels and $5-\mathrm{HT}_{2}$ receptors with nexopamil during myocardial ischemia and reperfusion in dogs: comparison to diltiazem," J. Cardiovasc. Pharmacol. 27, 777-787 (1996).

${ }^{35}$ C. F. Murphy, S. M. Horner, D. J. Dick, B. Coen, and M. J. Lab, “Electrical alternans and the onset of rate-induced pulsus alternans during acute regional ischaemia in the anaesthetised pig heart," Cardiovasc. Res. 32, 138-147 (1996).

${ }^{36} \mathrm{H}$. Tachibana, I. Kubota, M. Yamaki, T. Watanabe, and H. Tomoike, "Discordant S-T alternans contributes to formation of reentry: a possible mechanism of reperfusion arrhythmia," Am. J. Physiol. 275, H116-H121 (1998).

${ }^{37}$ D. R. Adam, J. M. Smith, S. Akselrod, S. Nyberg, A. O. Powell, and R. J. Cohen, "Fluctuations in T-wave morphology and susceptibility to ventricular fibrillation," J. Electrocardiol. 17, 209-218 (1984)

${ }^{38}$ M. R. Guevara, "Chaotic cardiac dynamics," Doctoral thesis, McGill University, Montreal, 1984.

${ }^{39}$ D. S. Rosenbaum, P. Albrecht, and R. J. Cohen, "Predicting sudden cardiac death from $T$ wave alternans of the surface electrocardiogram: promise and pitfalls," J. Cardiovasc. Electrophysiol. 7, 1095-1111 (1996).

${ }^{40}$ S. H. Hohnloser, T. Klingenheben, Y.-G. Li, M. Zabel, J. Peetermans, and R. J. Cohen, "T wave alternans as a predictor of recurrent ventricular tachyarrhythmias in ICD recipients: prospective comparison with conventional risk markers,"' J. Cardiovasc. Electrophysiol. 9, 1258-1268 (1998).

${ }^{41} \mathrm{CH} 2000$ system, Cambridge Heart Inc., Cambridge, http:// www.cambridgeheart.com/camh/professional/index.html.

${ }^{42}$ M. R. Guevara, L. Glass, and A. Shrier, "Phase locking, period-doubling bifurcations, and irregular dynamics in periodically stimulated cardiac cells," Science 214, 1350-1353 (1981).

${ }^{43}$ M. R. Guevara, G. Ward, A. Shrier, and L. Glass, "Electrical alternans and period-doubling bifurcations," Computers in Cardiology (IEEE Comput. Soc. Press, Silver Spring, MD, 1984), pp. 167-170.

${ }^{44}$ A. L. Ritzenberg, D. R. Adam, and R. J. Cohen, "Period multiplyingevidence for nonlinear behaviour of the canine heart," Nature (London) 307, 159-161 (1984).

${ }^{45}$ G. V. Savino, L. Romanelli, D. L. González, O. Piro, and M. E. Val- entinuzzi, "Evidence for chaotic behavior in driven ventricles," Biophys. J. 56, 273-280 (1989).

${ }^{46}$ D. R. Chialvo, R. F. Gilmour, Jr., and J. Jalife, "Low dimensional chaos in cardiac tissue," Nature (London) 343, 653-657 (1990).

${ }^{47}$ M. R. Guevara, A. Shrier, and L. Glass, "Chaotic and complex cardiac rhythms," in Cardiac Electrophysiology: From Cell to Bedside, 1st ed., edited by D. P. Zipes and J. Jalife (Saunders, Philadelphia, 1990), pp. 192-201.

${ }^{48}$ M. R. Guevara and A. Shrier, "Rhythms produced by high-amplitude periodic stimulation of spontaneously beating aggregates of embryonic chick ventricular myocytes," Ann. N. Y. Acad. Sci. 591, 11-22 (1990).

${ }^{49}$ J. Sun, F. Amellal, L. Glass, and J. Billette, "Alternans and perioddoubling bifurcations in atrioventricular nodal conduction," J. Theor. Biol. 173, 79-91 (1995).

${ }^{50}$ R. F. Gilmour, Jr., N. F. Otani, and M. A. Watanabe, "Memory and complex dynamics in cardiac Purkinje fibers," Am. J. Physiol. 272, H1826-H1832 (1997).

${ }^{51}$ K. Hall, D. J. Christini, M. Tremblay, J. J. Collins, L. Glass, and J. Billette, "Dynamic control of cardiac alternans," Phys. Rev. Lett. 78, 45184521 (1997).

${ }^{52}$ A. L. Goldberger, V. Bhargava, B. J. West, and A. J. Mandell, "Some observations on the question: is ventricular fibrillation "chaos"?," Physica D 19, 282-289 (1986).

${ }^{53}$ D. T. Kaplan and R. J. Cohen, "Is fibrillation chaos?," Circ. Res. 67, 886-892 (1990)

${ }^{54}$ F. X. Witkowski, K. M. Kavanagh, P. A. Penkoske, R. Plonsey, M. L. Spano, W. L. Ditto, and D. T. Kaplan, "Evidence for determinism in ventricular fibrillation," Phys. Rev. Lett. 75, 1230-1233 (1995).

${ }^{55}$ P. V. Bayly, B. H. KenKnight, J. M. Rogers, E. E. Johnson, R. E. Ideker, and W. M. Smith, "Spatial organization, predictability, and determinism in ventricular fibrillation," Chaos 8, 103-115 (1998).

${ }^{56}$ J. N. Weiss, A. Garfinkel, H. S. Karagueuzian, Z. Qu, and P.-S. Chen, "Chaos and the transition to ventricular fibrillation. A new approach to antiarrhythmic drug evaluation," Circulation 99, 1819-2826 (1999).

${ }^{57}$ A. V. Panfilov and A. V. Holden, "Spatiotemporal irregularity in a twodimensional model of cardiac tissue," Int. J. Bifurcation Chaos Appl. Sci. Eng. 1, 219-225 (1991)

${ }^{58}$ A. Karma, "Electrical alternans and spiral wave breakup in cardiac tissue," Chaos 4, 461-472 (1994).

${ }^{59} \mathrm{M}$. Courtemanche, "Complex spiral wave dynamics in a spatially distributed ionic model of cardiac electrical activity," Chaos 6, 579-600 (1996).

${ }^{60}$ F. Fenton and A. Karma, "Vortex dynamics in three-dimensional continuous myocardium with fiber rotation: filament instability and fibrillation," Chaos 8, 20-47 (1998).

${ }^{61} \mathrm{Z}$. Qu, J. N. Weiss, and A. Garfinkel, "Cardiac electrical restitution properties and stability of reentrant spiral waves: a simulation study," Am. J. Physiol. 276, H269-H283 (1999)

${ }^{62}$ A. L. Wit and M. J. Janse, The Ventricular Arrhythmias of Ischemia and Infarction. Electrophysiological Mechanisms (Futura, Mount Kisco, 1993).

${ }^{63}$ H. S. Karagueuzian and W. J. Mandel, "Electrophysiologic mechanisms of ischemic ventricular arrhythmias: experimental and clinical correlations," in Cardiac Arrhythmias, 3rd ed., edited by W. J. Mandel (Lippincott, Philadelphia, 1995), pp. 563-603.

${ }^{64} \mathrm{C}$. H. Orchard and H. E. Cingolani, " Acidosis and arrhythmias in cardiac muscle," Cardiovasc. Res. 28, 1312-1319 (1994).

${ }^{65}$ R. Coronel, J. W. T. Fiolet, F. J. G. Wilms-Schopman, T. Opthof, A. F. M. Schaapherder, and M. J. Janse, "Distribution of extracellular potassium and electrophysiologic changes during two-stage coronary ligation in the isolated, perfused canine heart," Circulation 80, 165-177 (1989).

${ }^{66}$ R. Coronel, F. J. G. Wilms-Schopman, L. R. C. Dekker, and M. J. Janse, "Heterogeneities in $\left[\mathrm{K}^{+}\right]_{0}$ and TQ potential and the inducibility of ventricular fibrillation during acute regional ischemia in the isolated perfused porcine heart," Circulation 92, 120-129 (1995).

${ }^{67}$ R. Coronel, J. W. T. Fiolet, F. J. G. Wilms-Schopman, A. F. M. Schaapherder, T. A. Johnson, L. S. Gettes, and M. J. Janse, "Distribution of extracellular potassium and its relation to electrophysiologic changes during acute myocardial ischemia in the isolated perfused porcine heart," Circulation 77, 1125-1138 (1988).

${ }^{68}$ H. Moréna, M. J. Janse, J. W. T. Fiolet, W. J. G. Krieger, H. Crijns, and D. Durrer, "Comparison of the effects of regional ischemia, hypoxia, hyperkalemia, and acidosis on intracellular and extracellular potentials and metabolism in the isolated porcine heart," Circ. Res. 46, 634-656 (1980) 
${ }^{69}$ M. J. Curtis, "The rabbit dual coronary perfusion model: a new method for assessing the pathological relevance of individual products of the ischaemic milieu: role of potassium in arrhythmogenesis," Cardiovasc. Res. 25, 1010-1022 (1991).

${ }^{70} \mathrm{~A}$. Xu and M. R. Guevara, "Two forms of spiral-wave reentry in an ionic model of ischemic ventricular myocardium,' Chaos 8, 157-174 (1998).

${ }^{71}$ S. Dokos, B. G. Celler, and N. H. Lovell, "'Modification of DiFrancescoNoble equations to simulate the effects of vagal stimulation on in vivo mammalian sinoatrial node electrical activity,' Ann. Biomed. Eng. 21, 321-335 (1993)

${ }^{72}$ A. Varghese and R. L. Winslow, "Dynamics of the calcium subsystem in cardiac Purkinje fibers," Physica D 68, 364-386 (1993).

${ }^{73}$ A. Varghese and R. L. Winslow, "Dynamics of abnormal pacemaking activity in cardiac Purkinje fibers,'’ J. Theor. Biol. 168, 407-420 (1994).

${ }^{74} \mathrm{~S}$. Guan, Q. Lu, and K. Huang, "A discussion about the DiFrancescoNoble model,' J. Theor. Biol. 189, 27-32 (1997).

${ }^{75}$ A. Varghese and G. R. Sell, "A conservation principle and its effect on the formulation of $\mathrm{Na}-\mathrm{Ca}$ exchanger current in cardiac cells," J. Theor. Biol. 189, 33-40 (1997)

${ }^{76}$ C.-h. Luo and Y. Rudy, "A model of the ventricular cardiac action potential. Depolarization, repolarization, and their interaction," Circ. Res. 68, 1501-1526 (1991)

${ }^{77} \mathrm{G}$. Isenberg and U. Klöckner, "Calcium currents of isolated bovine ventricular myocytes are fast and of large amplitude,' Pflüg. Arch. 395, 30-41 (1982).

${ }^{78}$ M. R. Franz, C. D. Swerdlow, L. B. Liem, and J. Schaefer, "Cycle length dependence of human action potential duration in vivo,' J. Clin. Invest. 82, 972-979 (1988)

${ }^{79}$ P. Taggart, P. M. I. Sutton, M. R. Boyett, M. Lab, and H. Swanton, "Human ventricular action potential duration during short and long cycles. Rapid modulation by ischemia,' Circulation 94, 2526-2534 (1996).

${ }^{80}$ B. S. Koller, P. E. Karasik, A. J. Solomon, and M. R. Franz, "Relation between repolarization and refractoriness during programmed electrical stimulation in the human right ventricle. Implications for ventricular tachycardia induction,' Circulation 91, 2378-2384 (1995).

${ }^{81}$ N. El-Sherif, B. J. Scherlag, and R. Lazzara, "Electrode catheter recordings during malignant ventricular arrhythmia following experimental acute myocardial ischemia. Evidence for re-entry due to conduction delay and block in ischemic myocardium,' Circulation 51, 1003-1014 (1975).

${ }^{82}$ N. El-Sherif, B. J. Scherlag, R. Lazzara, and R. R. Hope, "Re-entrant ventricular arrhythmias in the late myocardial infarction period. 1. Conduction characteristics in the infarction zone,' Circulation 55, 686-702 (1977).

${ }^{83}$ A. R. Yehia, D. Jeandupeux, F. Alonso, and M. R. Guevara, "Hysteresis and bistability in the direct transition from $1: 1$ to $2: 1$ rhythm in periodically driven single ventricular cells," Chaos 9, 916-931 (1999).

${ }^{84}$ M. Delmar, D. C. Michaels, and J. Jalife, "Slow recovery of excitability and the Wenckebach phenomenon in the single guinea pig ventricular myocyte,' Circ. Res. 65, 761-774 (1989).

${ }^{85}$ M. R. Guevara, D. Jeandupeux, F. Alonso, and N. Morissette, "Wenckebach rhythms in isolated ventricular heart cells," in International Conference on Singular Behavior and Nonlinear Dynamics, edited by St. Pnevmatikos, T. Bountis, and Sp. Pnevmatikos (World Scientific, Singapore, 1989), Vol. 2, pp. 629-642.

${ }^{86}$ A. R. Yehia, A. Shrier, K. C. -L. Lo, and M. R. Guevara, "Transient outward current contributes to Wenckebach-like rhythms in isolated rabbit ventricular cells,', Am. J. Physiol. 273, H1-H11 (1997).

${ }^{87}$ M. R. Guevara, F. Alonso, D. Jeandupeux, and A. C. G. van Ginneken, "Alternans in periodically stimulated isolated ventricular myocytes: Experiment and model,' in Cell to Cell Signalling: From Experiments to Theoretical Models, edited by A. Goldbeter (Harcourt Brace Jovanovich, London, 1989), pp. 551-563.

${ }^{88} \mathrm{~J}$. Hescheler and R. Speicher, "Regular and chaotic behaviour of cardiac cells stimulated at frequencies between 2 and $20 \mathrm{~Hz}$,' Eur. Biophys. J. 17, 273-280 (1989).

${ }^{89}$ M. R. Guevara and L. Glass, "Phase locking, period doubling bifurcations and chaos in a mathematical model of a periodically driven oscillator: a theory for the entrainment of biological oscillators and the generation of cardiac dysrhythmias,' J. Math. Biol. 14, 1-23 (1982).

90 Y. Horikawa, "Period-doubling bifurcations and chaos in the decremental propagation of a spike train in excitable media,' Phys. Rev. E 50, 1708-1710 (1994)

${ }^{91}$ D. T. Kaplan, J. R. Clay, T. Manning, L. Glass, M. R. Guevara, and A.
Shrier, "Subthreshold dynamics in periodically stimulated squid giant axons,' Phys. Rev. Lett. 76, 4074-4077 (1996).

${ }^{92}$ Y. Horikawa, "Bifurcations in the decremental propagation of a spike train in the Hodgkin-Huxley model of low excitability," Biol. Cybern. 79, 251-261 (1998).

${ }^{93}$ R. M. Shaw and Y. Rudy, "Electrophysiological effects of acute myocardial ischemia. A mechanistic investigation of action potential conduction and conduction failure,' Circ. Res. 80, 124-138 (1997).

${ }^{94}$ V. G. Fast and A. G. Kléber, "Role of wavefront curvature in propagation of cardiac impulse,' Cardiovasc. Res. 33, 258-271 (1997).

${ }^{95}$ C. H. Orchard, E. McCall, M. S. Kirby, and M. R. Boyett, "Mechanical alternans during acidosis in ferret heart muscle," Circ. Res. 68, 69-76 (1991).

${ }^{96}$ D. S. Rubenstein and S. L. Lipsius, "Premature beats elicit a phase reversal of mechanoelectrical alternans in cat ventricular myocytes: A possible mechanism for reentrant arrhythmias,' Circulation 91, 201-214 (1995).

${ }^{97}$ A. Vinet, D. R. Chialvo, D. C. Michaels, and J. Jalife, "Nonlinear dynamics of rate-dependent activation in models of single cardiac cells," Circ. Res. 67, 1510-1524 (1990)

${ }^{98}$ A. Vinet and F. A. Roberge, "Excitability and repolarization in an ionic model of the cardiac cell membrane," J. Theor. Biol. 170, 183-199 (1994).

${ }^{99}$ M. R. Guevara, "Spatiotemporal patterns of block in an ionic model of cardiac Purkinje fibre," in From Chemical to Biological Organization, edited by M. Markus, S. C. Müller, and G. Nicolis (Springer-Verlag, Berlin, 1988), pp. 273-281.

${ }^{100}$ D. T. Kaplan. "The dynamics of cardiac electrical instability,' Doctoral thesis, Harvard, Cambridge, 1989.

${ }^{101}$ M. R. Boyett and B. R. Jewell, "A study of the factors responsible for rate-dependent shortening of the action potential in mammalian ventricular muscle,', J. Physiol. (London) 285, 359-380 (1978).

${ }^{102}$ H. Saitoh, J. C. Bailey, and B. Surawicz, "Alternans of action potential duration after abrupt shortening of cycle length: differences between dog Purkinje and ventricular muscle fibers," Circ. Res. 62, 1027-1040 (1988).

${ }^{103}$ H. Saitoh, J. C. Bailey, and B. Surawicz, "Action potential duration alternans in dog Purkinje and ventricular muscle fibers. Further evidence in support of two different mechanisms," Circulation 80, 1421-1431 (1989).

${ }^{104}$ M. L. Koller, M. L. Riccio, and R. F. Gilmour, Jr., “'Dynamic restitution of action potential duration during electrical alternans and ventricular fibrillation,', Am. J. Physiol. 275, H1635-H1642 (1998).

${ }^{105}$ J. M. Pastore, S. D. Girouard, K. R. Laurita, F. G. Akar, and D. S. Rosenbaum, "Mechanism linking T-wave alternans to the genesis of cardiac fibrillation," Circulation 99, 1385-1394 (1999).

${ }^{106}$ M. L. Riccio, M. L. Koller, and R. F. Gilmour, Jr., 'Electrical restitution and spatiotemporal organization during ventricular fibrillation,' Circ. Res. 84, 955-963 (1999).

${ }^{107}$ J. B. Nolasco and R. W. Dahlen, "A graphic method for the study of alternation in cardiac action potentials,'’ J. Appl. Physiol. 25, 191-196 (1968).

${ }^{108}$ T. J. Lewis and M. R. Guevara, "Chaotic dynamics in an ionic model of the propagated cardiac action potential,' J. Theor. Biol. 146, 407-432 (1990).

${ }^{109}$ N. F. Otani and R. F. Gilmour, Jr., "'Memory models for the electrical properties of local cardiac systems," J. Theor. Biol. 187, 409-436 (1997).

${ }^{110}$ W. H. Gaskell, "On the rhythm of the heart of the frog, and on the nature of the action of the vagus nerve,', Philos. Trans. R. Soc. London 173, 993-1033 (1882).

${ }^{111}$ J. M. Smith and R. J. Cohen, "Simple finite-element model accounts for wide range of cardiac dysrhythmias,' Proc. Natl. Acad. Sci. USA 81, 233-237 (1984).

${ }^{112}$ R. W. Kurz, X. L. Ren, and M. R. Franz, "Dispersion and delay of electrical restitution in the globally ischaemic heart,' 'Eur. Heart J. 15, 547-554 (1994).

${ }^{113}$ J.-M. Cao, Z. Qu, Y.-H. Kim, T.-J. Wu, A. Garfinkel, J. N. Weiss, H. S. Karagueuzian, and P.-S. Chen, "Spatiotemporal heterogeneity in the induction of ventricular fibrillation by rapid pacing. Importance of cardiac restitution properties,' Circ. Res. 84, 1318-1331 (1999).

${ }^{114}$ H.-C. Lee, R. Mohabir, N. Smith, M. R. Franz, and W. T. Clusin, "Effect of ischemia on calcium-dependent fluorescence transients in rabbit hearts containing Indo 1. Correlation with monophasic action potentials and contraction," Circulation 78, 1047-1059 (1988). 
${ }^{115}$ Y. Kihara and J. P. Morgan, "Abnormal $\mathrm{Ca}_{\mathrm{i}}^{2+}$ handling is the primary cause of mechanical alternans: study in ferret ventricular muscles,' Am. J. Physiol. 261, H1746-H1755 (1991).

${ }^{116}$ Y. Hirayama, H. Saitoh, H. Atarashi, and H. Hayakawa, "Electrical and mechanical alternans in canine myocardium in vivo. Dependence on intracellular calcium cycling,', Circulation 88, 2894-2901 (1993).

${ }^{117} \mathrm{Y}$. Wu and W. T. Clusin, "Calcium transient alternans in blood-perfused ischemic hearts: observations with fluorescent indicator Fura Red,' Am. J. Physiol. 273, H2161-H2169 (1997).

${ }^{118}$ W. Shimizu and C. Antzelevitch, "Cellular and ionic basis for T-wave alternans under long-QT conditions,'” Circulation 99, 1499-1507 (1999).

${ }^{119}$ M. J. Lab and J. A. Lee, "Changes in intracellular calcium during mechanical alternans in isolated ferret ventricular muscle," Circ. Res. 66, 585-595 (1990).

${ }^{120}$ Y. Hirata, I. Kodama, N. Iwamura, T. Shimizu, J. Toyama, and K. Yamada, "Effects of verapamil on canine Purkinje fibres and ventricular muscle fibres with particular reference to the alternation of action potential duration after a sudden increase in driving rate,' Cardiovasc. Res. 13, 1-8 (1979).

${ }^{121}$ H. Hashimoto, K. Suzuki, S. Miyake, and M. Nakashima, "Effect of calcium antagonists on the electrical alternans of the ST segment during acute coronary occlusion in dogs,'” Jpn. Heart J. 22, 247-256 (1981).

${ }^{122}$ H. Hashimoto, K. Suzuki, S. Miyake, and M. Nakashima, "Effects of calcium antagonists on the electrical alternans of the ST segment and on associated mechanical alternans during acute coronary occlusion in dogs,', Circulation 68, 667-672 (1983).

${ }^{123}$ T. Hayakawa, Y. Nagamoto, K. Ninomiya, S. Abe, T. Fukumoto, and A. Kuroiwa, "Effects of heart rate and diltiazem hydrochloride on alternans of ST segment elevation and ventricular arrhythmia during acute myocardial ischaemia in dogs,', Cardiovasc. Res. 23, 520-528 (1989).

${ }^{124}$ A. Vinet and F. A. Roberge, "Analysis of an iterative difference equation model of the cardiac cell membrane,' J. Theor. Biol. 170, 201-214 (1994).

${ }^{125}$ G. M. Hall, S. Bahar, and D. J. Gauthier, "Prevalence of rate-dependent behaviors in cardiac muscle,', Phys. Rev. Lett. 82, 2995-2998 (1999).

${ }^{126}$ G. R. Mines, "On dynamic equilibrium in the heart,' J. Physiol. (London) 46, 349-383 (1913).

${ }^{127}$ E. Pruvot, A. de Torrente, G. M. de Ferrari, P. J. Schwartz, and J.-J. Goy, "Two-to-one AV block associated with the congenital long QT syndrome,' J. Cardiovasc. Electrophysiol. 10, 108-113 (1999).

${ }^{128}$ J. H. Jensen, P. L. Christiansen, and A. C. Scott, "Chaos in the BeelerReuter system for the action potential of ventricular myocardial fibres,', Physica D 13, 269-277 (1984).

${ }^{129}$ D. C. Michaels, D. R. Chialvo, E. P. Matyas, and J. Jalife, "Chaotic activity in a mathematical model of the vagally driven sinoatrial node,', Circ. Res. 65, 1350-1360 (1989).

${ }^{130} \mathrm{H}$. Zhang and A. V. Holden, "Chaotic meander of spiral waves in the
FitzHugh-Nagumo system," Chaos, Solitons and Fractals 5, 661-670 (1995).

${ }^{131}$ Z. Qu, J. N. Weiss, and A. Garfinkel, "Spatiotemporal chaos in a simulated ring of cardiac cells," Phys. Rev. Lett. 78, 1387-1390 (1999).

${ }^{132}$ A. V. Panfilov, "Spiral breakup as a model of ventricular fibrillation," Chaos 8, 57-64 (1998)

${ }^{133}$ M. C. Strain and H. S. Greenside, "Size-dependent transition to highdimensional chaotic dynamics in a two-dimensional excitable medium," Phys. Rev. Lett. 80, 2306-2309 (1998).

${ }^{134}$ I. Kodama, A. Wilde, M. J. Janse, D. Durrer, and K. Yamada, “Combined effects of hypoxia, hyperkalemia and acidosis on membrane action potential and excitability of guinea-pig ventricular muscle," J. Mol. Cell. Cardiol. 16, 247-259 (1984).

${ }^{135}$ M. Kleinfeld, E. Stein, and J. Magin, "Electrical alternans in single ventricular fibers of the frog heart," Am. J. Physiol. 187, 139-142 (1956).

${ }^{136}$ Y. Hirata, J. Toyama, and K. Yamada, "Effects of hypoxia or low pH on the alternation of canine ventricular action potentials following an abrupt increase in driving rate," Cardiovasc. Res. 14, 108-115 (1980).

${ }^{137}$ J. M. Ferrero, Jr., J. Sáiz, J. M. Ferrero, and N. V. Thakor, "Simulation of action potentials from metabolically impaired cardiac myocytes. Role of ATP-sensitive $\mathrm{K}^{+}$current,"' Circ. Res. 79, 208-221 (1996).

${ }^{138}$ R. M. Shaw and Y. Rudy, "Electrophysiological effects of acute myocardial ischemia: a theoretical study of altered cell excitability and action potential duration," Cardiovasc. Res. 35, 256-272 (1997).

${ }^{139}$ F. F. -T. Ch'en, R. D. Vaughan-Jones, K. Clarke, and D. Noble, "Modelling myocardial ischaemia and reperfusion,” Prog. Biophys. Mol. Biol. 69, 515-538 (1998).

${ }^{140}$ N. A. M. Estes III, G. Michaud, D. P. Zipes, N. El-Sherif, F. J. Venditti, D. S. Rosenbaum, P. Albrecht, P. J. Wang, and R. J. Cohen, "Electrical alternans during rest and exercise as predictors of vulnerability to ventricular arrhythmias,"' Am. J. Cardiol. 80, 1314-1318 (1997).

${ }^{141}$ J. Y. Wei, A. Genecin, H. L. Greene, and S. C. Achuff, "Coronary spasm with ventricular fibrillation during thyrotoxicosis: response to attaining euthyroid state," Am. J. Cardiol. 43, 335-339 (1979).

${ }^{142}$ P. Laguna, G. B. Moody, J. Garcia, A. L. Goldberger, and R. G. Mark, "Analysis of the ST-T complex of the electrocardiogram using the Karhunen-Loeve transform: adaptive monitoring and alternans detection," Med. Biol. Eng. Comput. 37, 175-189 (1999).

${ }^{143}$ N. El-Sherif, R. R. Hope, B. J. Scherlag, and R. Lazzara, "Re-entrant ventricular arrhythmias in the late myocardial infarction period. 2. Patterns of initiation and termination of re-entry," Circulation 55, 702-719 (1977).

${ }^{144}$ H. Tachibana, M. Yamaki, I. Kubota, T. Watanabe, S. Yamauchi, and H. Tomoike, "Intracoronary flecainide induces ST alternans and reentrant arrhythmia on intact canine heart. A role of 4-aminopyridine-sensitive current," Circulation 99, 1637-1643 (1999). 